\title{
Safety, pharmacokinetics and antiviral activity of PGT121, a broadly neutralizing monoclonal antibody against HIV-1: a randomized, placebo-controlled, phase 1 clinical trial
}

\author{
Kathryn E. Stephenson ${ }^{1,2,3,13}$, Boris Julg, ${ }^{1,3,43}$, C. Sabrina Tan ${ }^{1,2}$, Rebecca Zash ${ }^{1,2}$, Stephen R. Walsh (1) 1, \\ Charlotte-Paige Rolle ${ }^{5}$, Ana N. Monczor6, Sofia Lupo', Huub C. Gelderblom ${ }^{7}$, Jessica L. Ansel', \\ Diane G. Kanjilal', Lori F. Maxfield [1], Joseph Nkolola', Erica N. Borducchi', Peter Abbink ${ }^{1}$ ', \\ Jinyan Liu', Lauren Peter ${ }^{1}$ ', Abishek Chandrashekar ${ }^{\circledR 1}$, Ramya Nityanandam', Zijin Lin', \\ Alessandra Setaro', Joseph Sapiente ${ }^{1}$, Zhilin $\mathrm{Chen}^{3}$, Lisa Sunner7, Tyler Cassidy ${ }^{8}{ }^{8}$, Chelsey Bennett ${ }^{9}$, \\ Alicia Sato ${ }^{9}$, Bryan Mayer ${ }^{9}{ }^{9}$, Alan S. Perelson ${ }^{8}{ }^{8}$, Allan deCamp ${ }^{9}$, Frances H. Priddy ${ }^{7}$, Kshitij Wagh $^{8}$, \\ Elena E. Giorgi ${ }^{8}$, Nicole L. Yates ${ }^{10,11}$, Roberto C. Arduino ${ }^{6}$, Edwin DeJesus ${ }^{5}$, Georgia D. Tomaras ${ }^{10,11}$, \\ Michael S. Seaman', Bette Korber ${ }^{8,12}$ and Dan H. Barouch $\mathbb{1}^{1,2,3 凶}$
}

Human immunodeficiency virus (HIV)-1-specific broadly neutralizing monoclonal antibodies are currently under development to treat and prevent HIV-1 infection. We performed a single-center, randomized, double-blind, dose-escalation, placebo-controlled


and HIV-infected adults on antiretroviral therapy (ART), as well as a multicenter, open-label trial of one infusion of PGT121 at $30 \mathrm{mg} \mathrm{kg}^{-1}$ in viremic HIV-infected adults not on ART (no. NCT02960581). The primary endpoints were safety and tolerability, pharmacokinetics (PK) and antiviral activity in viremic HIV-infected adults not on ART. The secondary endpoints were changes in anti-PGT121 antibody titers and CD4 ${ }^{+}$- - -cell count, and development of HIV-1 sequence variations associated with PGT121 resistance. Among 48 participants enrolled, no treatment-related serious adverse events, potential immune-mediated diseases or Grade 3 or higher adverse events were reported. The most common reactions among PGT121 recipients were intravenous/injection site tenderness, pain and headache. Absolute and relative CD4 ${ }^{+}$T-cell counts did not change following PGT121 infusion in HIV-infected participants. Neutralizing anti-drug antibodies were not elicited. PGT121 reduced plasma HIV RNA levels by a median of $1.77 \mathrm{log}$ in viremic participants, with a viral load nadir at a median of 8.5 days. Two individuals with low baseline viral loads experienced ART-free viral suppression for $\geq \mathbf{1 6 8}$ days following antibody infusion, and rebound viruses in these individuals demonstrated full or partial PGT121 sensitivity. The trial met the prespecified endpoints. These data suggest that further investigation of the potential of antibody-based therapeutic strategies for long-term suppression of HIV is warranted, including in individuals off ART and with low viral load.

$\mathrm{H}$ uman immunodeficiency virus-1-specific broadly neutralizing monoclonal antibodies (bNAbs) that target the HIV-1 envelope (Env) protein are currently under development as potential tools to treat and prevent HIV-1 infection. Several bNAbs have been tested to date in HIV-1-infected individuals, including the CD4-binding, site-specific antibodies VRC01, 3BNC117, VRC07523LS and N6-LS, the V3-glycan-specific antibody 10-1074 and the V2-apex-specific antibodies PGDM1400 and CAP256-VRC26.25 (refs. ${ }^{1-8}$ ). These bNAbs (alone or in combination) have been shown to decrease plasma HIV RNA levels by $1-2 \log$ in viremic, HIV-1-infected participants not on $\mathrm{ART}^{1-4}$. In these studies, bNAb resistance emerged in nearly all treated participants who received monotherapy, but in two notable cases ${ }^{4}$ the repeated administration of a combination of two bNAbs in the setting of viremia maintained viral suppression for 3 months without development of resistance to either antibody. bNAbs have also been shown to delay viral rebound in HIV-1-infected participants on ART who were undergoing analytical treatment interruption ${ }^{6-8}$. However, it has not yet

${ }^{1}$ Center for Virology and Vaccine Research, Beth Israel Deaconess Medical Center, Boston, MA, USA. ${ }^{2}$ Division of Infectious Diseases, Beth Israel Deaconess Medical Center, Boston, MA, USA. ${ }^{3}$ Ragon Institute of MGH, MIT and Harvard, Cambridge, MA, USA. ${ }^{4}$ Infectious Disease Division, Massachusetts General Hospital, Boston, MA, USA. ${ }^{5}$ Orlando Immunology Center, Orlando, FL, USA. ${ }^{6}$ McGovern Medical School at The University of Texas Health Science Center, Houston, TX, USA. ${ }^{7}$ International AIDS Vaccine Initiative, New York, NY, USA. ${ }^{8}$ Theoretical Biology and Biophysics Group, Los Alamos National Laboratory, Los Alamos, NM, USA. ${ }^{9}$ Statistical Center for HIV/AIDS Research and Prevention, Fred Hutchinson Cancer Research Center, Seattle, WA, USA. ${ }^{10}$ Duke Human Vaccine Institute, Duke University, Durham, NC, USA. "Departments of Surgery, Immunology and Molecular Genetics and Microbiology, Duke University, Durham, NC, USA. ${ }^{12}$ New Mexico Consortium, Los Alamos, NM, USA. ${ }^{13}$ These authors contributed equally:

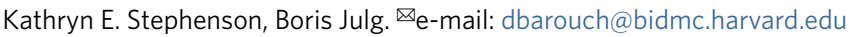




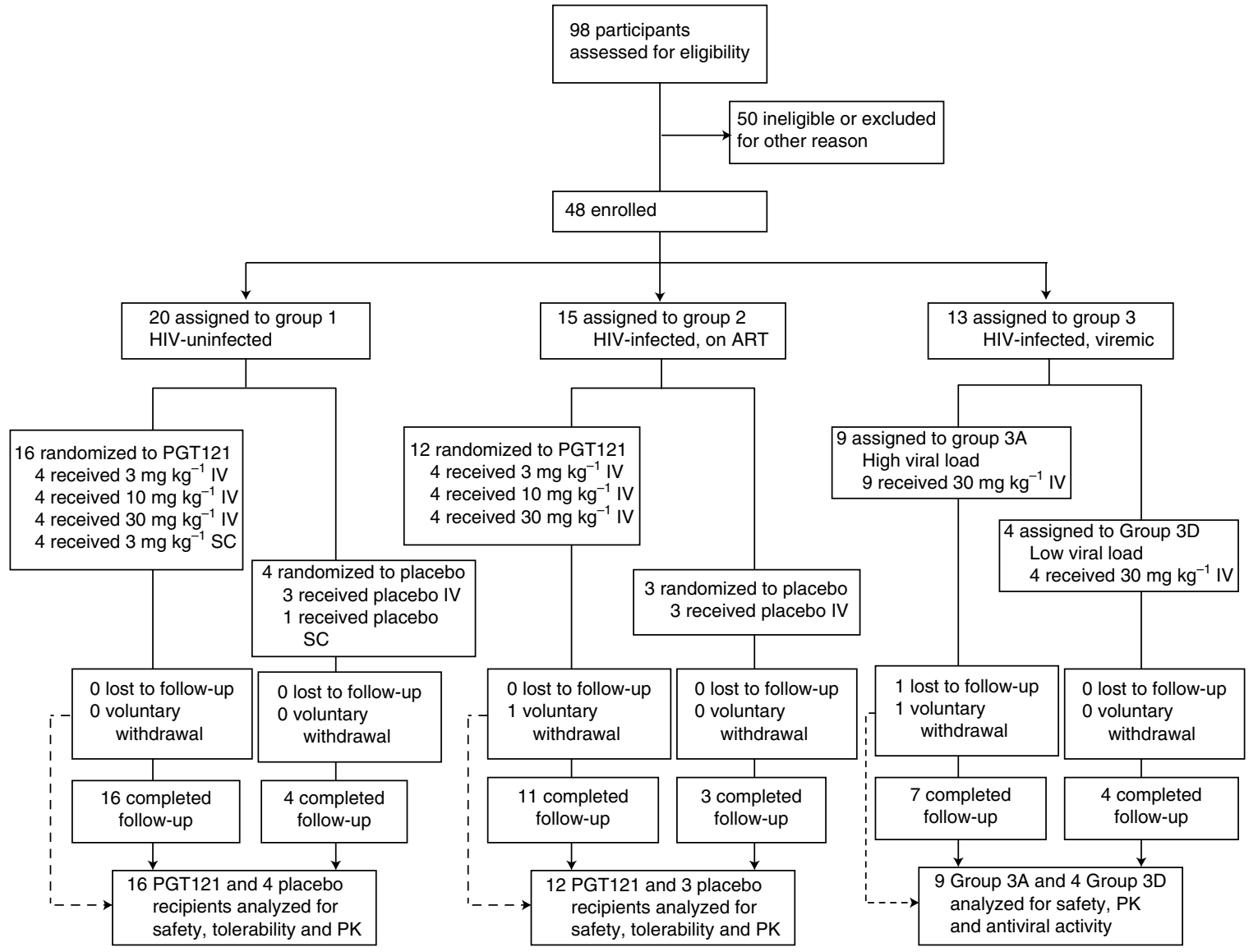

Fig. 1 | Trial profile. Participant recruitment, randomization, and follow up are depicted. Participants were enrolled concurrently at BIDMC (Beth Israel Deaconess Medical Center), OIC (Orlando Immunology Center), and HART (Houston AIDS Research Team, McGovern Medical School at The University of Texas Health Science Center).

been demonstrated whether a single bNAb given once can maintain viral suppression longer than 3 months without the development of resistance.

PGT121 is a monoclonal antibody isolated in 2011 from an African donor infected with HIV-1 subtype A, whose sera had demonstrated superior neutralization breadth and potency in an observational cohort, and so was considered an 'elite neutralizer'. PGT121 targets a V3-glycan-dependent site on HIV-1 Env'. Structural studies suggest that PGT121 inhibits gp120 binding to the CD4 receptor by interfering with Env receptor engagement and blocking viral entry ${ }^{10}$, and in vitro experiments demonstrated that PGT121 (ref. ${ }^{9}$ ) was able to neutralize $64 \%$ of 634 diverse tier 2 (for example, more difficult to neutralize) HIV-1 strains tested and recorded in the Los Alamos HIV database tool CATNAP ${ }^{11}$ (data available in Supplementary material), with a geometric mean half-maximal inhibitory concentration $\left(\mathrm{IC}_{50}\right)$ of $0.07 \mu \mathrm{g} \mathrm{ml}^{-1}$ among sensitive isolates. PGT121 decreased viral load (VL) in simian-human immunodeficiency virus (SHIV)-infected rhesus macaques ${ }^{12}$, delayed SHIV rebound following ART interruption when combined with a Toll-like receptor 7 agonist $^{13}$ and protected uninfected monkeys against SHIV challenge at a plasma concentration of $<5 \mu \mathrm{g} \mathrm{ml}^{-1}$ (refs. ${ }^{14,15}$ ).

Here we report the results of a multicenter phase 1 clinical trial testing the safety, PK, antiviral activity and evolutionary mechanisms of escape of PGT121 in viremic HIV-infected adults not on ART $(n=13)$, as well as safety and PK in HIV-uninfected adults $(n=20)$ and HIV-infected adults on ART $(n=15)$. In the first part of the study we evaluated three doses $\left(3,10\right.$ and $\left.30 \mathrm{mg} \mathrm{kg}^{-1}\right)$ and two routes (intravenous (IV) and subcutaneous (SC)) of PGT121 given once in HIV-uninfected adults and HIV-infected adults on ART; participants were randomized 4:1 to receive PGT121 or placebo. In the second part of the study, viremic HIV-infected adults not on ART received a single IV administration of PGT121 at $30 \mathrm{mg} \mathrm{kg}^{-1}$.

\section{Results}

Study population. Ninety-eight participants were screened, of whom 50 were found to be ineligible or were excluded for other reasons (Fig. 1). The first participant was enrolled on 17 January 2017 , the last on 22 January 2019 and the final study visit was completed on 8 July 2019. The most common reason for screen failure was unwillingness to comply with the protocol requirements (Supplementary Table 1). In Part 1 of the study, HIV-uninfected adults $(n=20)$ were enrolled in Group 1 and HIV-infected adults on ART $(n=15)$ in Group 2. After determination that PGT121 administered at $30 \mathrm{mg} \mathrm{kg}^{-1}$ was safe and well tolerated in Groups 1 and 2, Part 2 of the study was initiated with HIV-infected participants not on ART and viremic $(n=13)$ being enrolled in Group 3. As prespecified in the protocol, participants in Group 3 were assigned to one of two groups based on their screening plasma HIV RNA levels: nine participants were assigned to the high-viral-load group $\left(2,000-100,000\right.$ copies $\left.\mathrm{ml}^{-1}\right)$ and four to the low-viral-load group $\left(100-2,000\right.$ copies $\left.\mathrm{ml}^{-1}\right)$. Demographics of the study population are shown in Table 1 and Supplementary Table 2.

Safety and tolerability. No related serious adverse events (SAEs), potential immune-mediated diseases or Grade 3 or higher solicited or unsolicited adverse events (AEs) were reported during the study 
Table 1 | Study population

\begin{tabular}{|c|c|c|c|c|c|}
\hline \multirow[t]{2}{*}{ Parameter } & \multicolumn{2}{|c|}{ HIV-uninfected } & \multicolumn{2}{|c|}{ HIV-infected on ART } & \multirow{2}{*}{$\begin{array}{l}\text { HIV-infected viremic } \\
\text { Active }\end{array}$} \\
\hline & Active & Placebo & Active & Placebo & \\
\hline & $n=16$ & $n=4$ & $n=12$ & $n=3$ & $n=13$ \\
\hline Male, $n(\%)$ & $6(38)$ & $3(75)$ & $10(83)$ & $3(100)$ & $12(92)$ \\
\hline \multicolumn{3}{|c|}{ Years since HIV diagnosis, mean (range) } & $14(1-27)$ & $15(6-27)$ & $3(0-20)$ \\
\hline \multicolumn{3}{|c|}{ Absolute CD4+ T-cell count, median (IQR) } & $706(530-838)$ & $738(708-757)$ & $568(494-920)$ \\
\hline \multicolumn{2}{|c|}{ HIV-1 RNA (copies $\mathrm{ml}^{-1}$ ) on day 0 , median (IQR) } & \multicolumn{2}{|c|}{ Low VL, $n=4$} & & $270(185-550)$ \\
\hline
\end{tabular}

(Supplementary Tables 3-5). One Group 3 PGT121 recipient reported a pregnancy 17 weeks after study product infusion (day 119); pregnancy was confirmed. The participant initiated ART immediately and delivered a healthy baby, who will be followed for safety until the end of the first year of life. Local solicited symptoms were reported by $12 / 41$ (29\%) PGT121 recipients and 4/7 (57\%) placebo recipients. Systemic solicited symptoms were reported by 12/41 (29\%) PGT121 recipients and $2 / 7$ (29\%) placebo recipients. The most common reactions among active PGT121 participants were IV/injection site tenderness $(n=11 / 41(27 \%))$, pain $(n=7 / 41(17 \%))$ and headache $(n=8 / 41(20 \%))$. Absolute and relative CD4 ${ }^{+} \mathrm{T}$-cell counts did not change following PGT121 administration in HIV-infected participants in Groups 2 or 3 (Supplementary Table 6).

Pharmacokinetics. The median (minimum, maximum) PGT121 elimination half-life was $22(16,29)$ days for HIV-uninfected participants, $16(11,30)$ days for HIV-infected participants on ART and 14 $(8,19)$ days for HIV-infected participants off ART and viremic (Fig. $2 \mathrm{a}-\mathrm{c}$ and Supplementary Tables 7-9). HIV-uninfected participants had greater PGT121 exposure over time compared to HIV-infected participants who received the same doses (Supplementary Table 9). The more rapid clearance rate among HIV-infected participants was probably due to the so-called sink effect, in which PGT121-virus complexes are removed and taken out of circulation. Interestingly, PGT121 concentrations measured by binding (direct readout) and neutralizing (indirect functional readout) antibody assays using the viruses CNE30 and X2088_c9 displayed substantial concordance (Extended Data Fig. 1a,b).

Anti-drug antibodies. At baseline, samples from all participants were below the threshold for positivity and were therefore determined to be PGT121 anti-drug antibody (ADA) negative (Extended Data Fig. 2a). At the last available time point for each participant, all samples remained ADA negative with the exception of samples from two participants (PTID 7945 from Group 2, at $30 \mathrm{mg} \mathrm{kg}^{-1} \mathrm{dose}_{\text {, }}$ and 2305 from Group 3 ) that gave positive signals on day 168 and were confirmed by PGT121 competition assay (Extended Data Fig. 2a,b). For both individuals, positive ADA serum samples were analyzed (using their day 0 baseline samples as reference) in a functional ADA assay in which the participant's serum was incubated with titrated amounts of PGT121 in TZM.bl cells infected with a PGT121-sensitive HIV-1 pseudovirus to assess anti-PGT121 neutralizing activity (Extended Data Fig. 2c). ADA ID ${ }_{50}$ neutralization titers against PGT121 were below the assay limit of detection $(<20)$ for both samples, indicating that detected ADA were non-neutralizing.

Antiviral activity. Thirteen HIV-infected participants not on ART were enrolled, all with a HIV-1 viral load at screening of between 100 and $<2,000$ copies $\mathrm{ml}^{-1}$ (low-viral-load group, $n=4$ ) or between
2,000 and 100,000 copies $\mathrm{ml}^{-1}$ (high-viral-load group, $n=9$; Table 1 and Supplementary Table 10). HIV-1 pseudoviruses constructed from plasma single-genome amplification of circulating viruses were tested for bNAb sensitivity by TZM-bl assay at baseline, with 8 of 13 participants $(61.5 \%)$ demonstrating full sensitivity to PGT121 at baseline (Extended Data Fig. 3), consistent with in vitro predictions of $59 \%$ sensitivity against 159 clade B viruses (CATNAP data; Supplementary material). Of note, 6 of 13 participants demonstrated either complete or partial levels of resistance $\left(\mathrm{IC}_{50}>1 \mu \mathrm{g} \mathrm{ml}^{-1}\right)$ to at least two bNAbs of different epitope specificity at baseline, demonstrating the prevalence of multi-bNAb resistance during chronic infection (Extended Data Fig. 3).

In the high-viral-load group, the median viral load at day 0 was $21,040$ (interquartile range $(\mathrm{IQR})=9,660-28,990)$. Following a single IV infusion of PGT121 at $30 \mathrm{mg} \mathrm{kg}^{-1}$ (Fig. 3a), four participants with PGT121 sensitivity and high viral load at baseline showed a decrease in viral load of $1.28-2.14 \log$ (median $1.77 \mathrm{log}$ ), with a viral load nadir at $7-10$ days (median 8.5 days), before viral load increased back to baseline by day 28. Rebound viruses isolated at this time point demonstrated PGT121 resistance (Fig. 3b). The three participants with complete baseline PGT121 resistance had no change in viral load compared to baseline following PGT121 infusion, while participants with a mix of sensitive and relatively resistant viral variants had brief dips in viral load (Fig. 3a and Supplementary Table 10). Notably, baseline virus sensitivity to PGT121 significantly correlated with the maximum drop in viral load observed following infusion (Kendall's $\tau=-0.64, P=0.0023$ ), as well as with faster decline in viral load (Kendall's $\tau=0.60, P=0.0092$ ) (Extended Data Fig. $4 a, c)$. These correlations remained significant even when factoring in changing levels of PGT121 over time (Extended Data Fig. $4 b, d)^{16,17}$. On the other hand, time to viral rebound could not be fully explained by the degree of PGT121 resistance present in rebounding viruses (Extended Data Fig. 4e,f). This suggests that other, unmeasured, factors, such as the presence of minority resistant variants among baseline circulating or latent viruses, might drive the time to rebound.

In the low-viral-load group, the median viral load at day 0 was 270 copies $\mathrm{ml}^{-1} \quad(\mathrm{IQ}=185-550)$ and circulating viruses were all PGT121 sensitive (Fig. 3). Following a single IV infusion of PGT121 at $30 \mathrm{mg} \mathrm{kg}^{-1}$ (Fig. 3a), all four participants had a decrease in viral load to $<40$ copies $\mathrm{ml}^{-1}$ (lower limit of quantification (LLoQ) of assay) by day 7. Two participants (PTIDs 2936 and 4236) had viral rebound by day 28 , and rebound viruses were PGT121 resistant (Fig. $3 \mathrm{~b})$. However, in two participants (PTIDs 6113 and 1536) viral load stayed suppressed at $<40$ copies $\mathrm{ml}^{-1}$ until rebound was detected at $\geq 168$ days (Fig. $3 \mathrm{c}$ ).

For both participants who exhibited long-term virologic control (PTIDs 6113 and 1536), viral rebound did not occur until PGT121 serum levels were below the limit of detection, suggesting substantial viral control even at very low antibody levels. For participant 



Fig. 2 | PGT121 PK. a-c, The PK profile of serum PGT121 is shown for HIV-uninfected participants (a), HIV-infected participants on antiretroviral therapy (b) and HIV-infected viremic participants (c).

6113, viral rebound was observed on day 168 and no PGT121 resistance was detected among rebound viruses (Fig. 3b). For participant 1536, viral rebound was not detected until the participant was brought back for a long-term extension of the study, at day 252 (Fig. 3b). Rebound viruses isolated from participant 1536 at that time point had a mixture of sensitivities: $7 / 10$ were resistant while $3 / 10$ remained sensitive (Fig. $3 \mathrm{~b}$ ). Emtricitabine and lamivudine drug levels of participants 6113 and 1536 were obtained at visit 11 (day 84), and both antiretroviral drug levels were below the limit of quantitation (note, these antiretrovirals were chosen for testing because either emtricitabine or lamivudine was present in nearly all available combination ART regimens at that time).
Pathways to PGT121 resistance. There were several distinct viral evolutionary patterns associated with the emergence of PGT121 resistance following treatment. Three participants $(2305,7190$ and 2936) demonstrated selection of a single resistant sequence variant, which emerged in a single lineage from more diverse baseline sequences (Extended Data Figs. 5 and 6). In these participants, loss of glycan N332 was associated with complete resistance to PGT121, consistent with previous studies ${ }^{18}$. For all other participants, PGT121 resistance was associated with more complex patterns of viral escape involving multiple resistance mutations (Extended Data Figs. 3 and 5-8) and multiple phylogenetic lineages (Extended Data Fig. 5). Resistance was mainly conferred by removal of the glycan at 332 (mutations at 332 and/or 334) but, in a few cases, also by mutations at site 325 in the GDIR motif located within Env V3 loop positions 324-327, a region critical for binding to the coreceptor CCR5 (refs. ${ }^{19,20}$ ) as well as V3 glycan bNAbs, and a well-established PGT121 resistance site ${ }^{18}$. For example in participants 2990 and 4236, PGT121-resistant viruses had Asn at 325 (D325N) and Lys at 325 (D325K), respectively, while retaining the glycan at N332. Rebound viruses from participants 6292 and 7190 showed resistance mutations at both 332/334 and 325. For participant 2319, a resistance pattern was observed where one amino acid change introduced a glycan at position N413 (Extended Data Figs. 5 and 6). The close structural proximity to glycan N33220 suggests that this modification is a candidate for driving of PGT121 resistance.

Eleven (11) of the 13 viremic participants had evidence of recombination in rebound viruses and, in two of these, recombination was shown to impact the acquisition of resistance to PGT121. Participant 6292 had highly diverse resistance signatures following PGT121 treatment, with multiple escape variants in both the D325 site (D to N, E or K) and the glycosylation sequon at N332 (NIS to either DIS or NIN), and different combinations of resistance mutations at the two sites were found. Most of the complex resistance forms in this participant were recombinants (Extended Data Fig. 7a). Participant 2990 had a single resistance mutation that emerged after PGT121 treatment, D325N. All of the viruses sampled following treatment carried $\mathrm{D} 325 \mathrm{~N}$ and were identified as recombinants. These viruses were relatively diverse, suggesting that the resistance mutation was moving through the quasispecies via recombination (Extended Data Fig. 7b).

We sequenced viruses from the two patients with long-term, antibiotic-mediated suppression at very late time points after virus rebound, at day 252 and beyond (Fig. 3a and Extended Data Fig. $6 c)$. For participant 1536, some rebound viruses retained sensitivity to PGT121 while others were completely resistant; the phylogenetic tree suggests that the re-emerging sensitive forms were very closely related to a baseline lineage (Extended Data Fig. 5). Viruses from participant 6113 were sensitive both before and after treatment (Fig. 3b).

While for most participants PGT121 resistance was accompanied by 10-1074 resistance, for participants 2990 and 2319, PGT121-resistant viruses remained highly sensitive to 10-1074 (Extended Data Fig. 3). Only one participant acquired resistance to a neutralizing antibody with an unrelated target, either PGDM1400 (a V2 antibody) or 3BNC117 (a CD4bs antibody), following administration of PGT121 (a V3 antibody) (Extended Data Fig. 3). Participant 2319 developed full resistance to PGDM1400, which was probably carried along when the virus selected for PGT121 resistance during treatment.

Building on previously established methods ${ }^{21,22}$, we developed a viral dynamic model for bNAb-mediated control of HIV-1 infection and fit it to viral load data from viremic patients in this study. Our model accurately fits the viral load data and makes predictions about the temporal dynamics of sensitive and resistant populations (Extended Data Fig. 8). For example, in some participants, such as 2305,2936 and 2990, the model predicted that a resistant population 

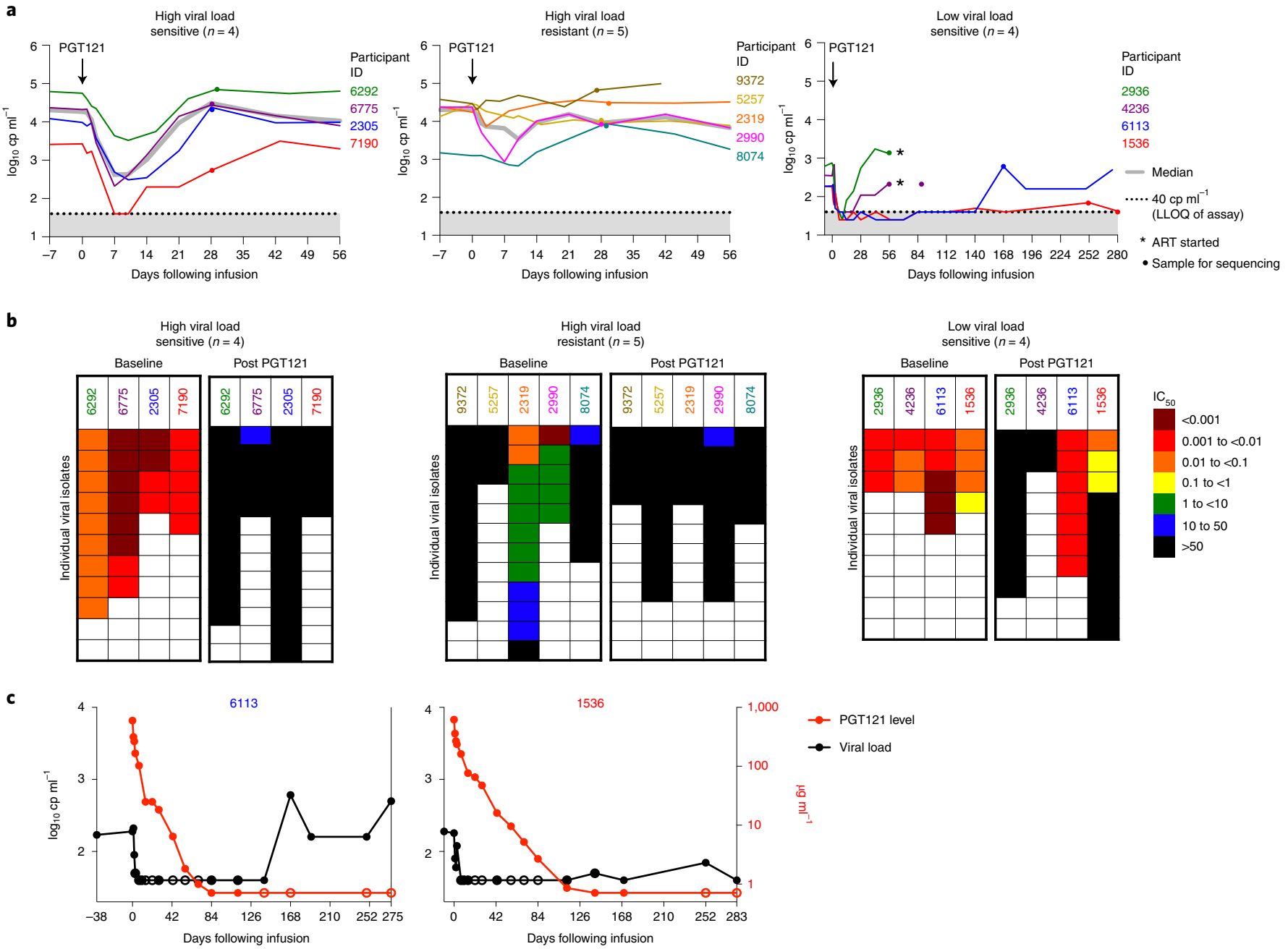

Fig. 3 | HIV-1 RNA viral load and sensitivity to PGT121 before and after PGT121 infusion. a, Plasma HIV-1 RNA levels are shown following PGT121 infusion in HIV-infected viremic participants not on ART. The arrow indicates the day of PGT121 infusion, the gray line equals median value and the dotted line indicates the lower limit of quantification for HIV-1 RNA levels (40 copies (cp) $\mathrm{ml}^{-1}$ ). Asterisks indicate the time point at which ART was started, and dots indicate when samples were collected for sequencing. $\mathbf{b}, I_{50}$ neutralization scores ( $\mu g \mathrm{ml}^{-1}$ ), indicating sensitivity or resistance to $\mathrm{PGT121}$ among isolated viruses, are shown for each participant, before and after PGT121 administration. c, HIV-1 viral load and PGT121 serum levels are shown for participants 6113 and 1536 following PGT121 infusion. Open circles indicate that either HIV-1 RNA (black) or PGT121 level (red) was below the limit of detection.

pre-exists, drives viral load rebound back to baseline and persists as the dominant population until the end of the observation period at day 56 (Extended Data Fig. 8). For both long-term controllers, 1536 and 6113, the model predicts that both sensitive and resistant populations are exquisitely controlled by PGT121 initially, and that the loss of control is due to an increase in the resistant population as antibody pressure wanes. However, ultimately the resistant population is replaced by a PGT121-sensitive population, possibly due to the lower replicative fitness of the resistant population (note, replicative fitness of isolated viruses was not tested in this study). The replacement by PGT121-sensitive virus after decay of PGT121 in serum was also seen for patient 6292 .

Immune responses. Recent data in rhesus macaque and human studies suggest that bNAb administration can enhance the autologous HIV-specific T-cell response ${ }^{23,24}$. To explore whether this potential vaccinal effect may have contributed to the long-term, antibiotic-mediated suppression observed for participants 6113 and 1536, we evaluated HIV-1-specific T-cell magnitude, breadth and functionality at baseline and at day 84 . We found no increase in the total number of Env-, Gag- or Pol-specific interferon-gamma
(IFN- $\gamma$ )- or tumor necrosis factor-alpha (TNF- $\alpha$-secreting T cells following infusion (Fig. 4a). Furthermore, no expansion of T-cell breadth (Fig. 4b) - as determined by the number of IFN- $\gamma$-secreting T cells recognizing peptide subpools across Gag and Pol proteinswas observed. We also investigated whether PGT121 had any immunomodulatory effect in the group of HIV-infected participants on ART with stably suppressed viral load (Group 2). However, we were unable to detect any major changes in Gag-, Pol- and Env-specific IFN- $\gamma$-, IL-2- or TNF- $\alpha$-secreting CD4 or CD8 T cells, or frequencies of activated $\mathrm{CD} 38^{+} \mathrm{Ki} 67^{+}$or exhausted $\mathrm{PD}$-1-expressing $\mathrm{CD} 4$ and CD8 Tcells specifically when compared to placebo control individuals (Extended Data Fig. 9). These data therefore support the conclusion that the viral control observed was mediated by bNAb alone and was not likely to have been supported by improved T-cell immunity following treatment.

\section{Discussion}

In this study, we demonstrate that the bNAb PGT121 potently and transiently inhibited HIV-1 RNA replication in viremic, HIV-infected individuals who had PGT121-sensitive viruses at baseline. Following a single IV infusion of PGT121 at $30 \mathrm{mg} \mathrm{kg}^{-1}$, 

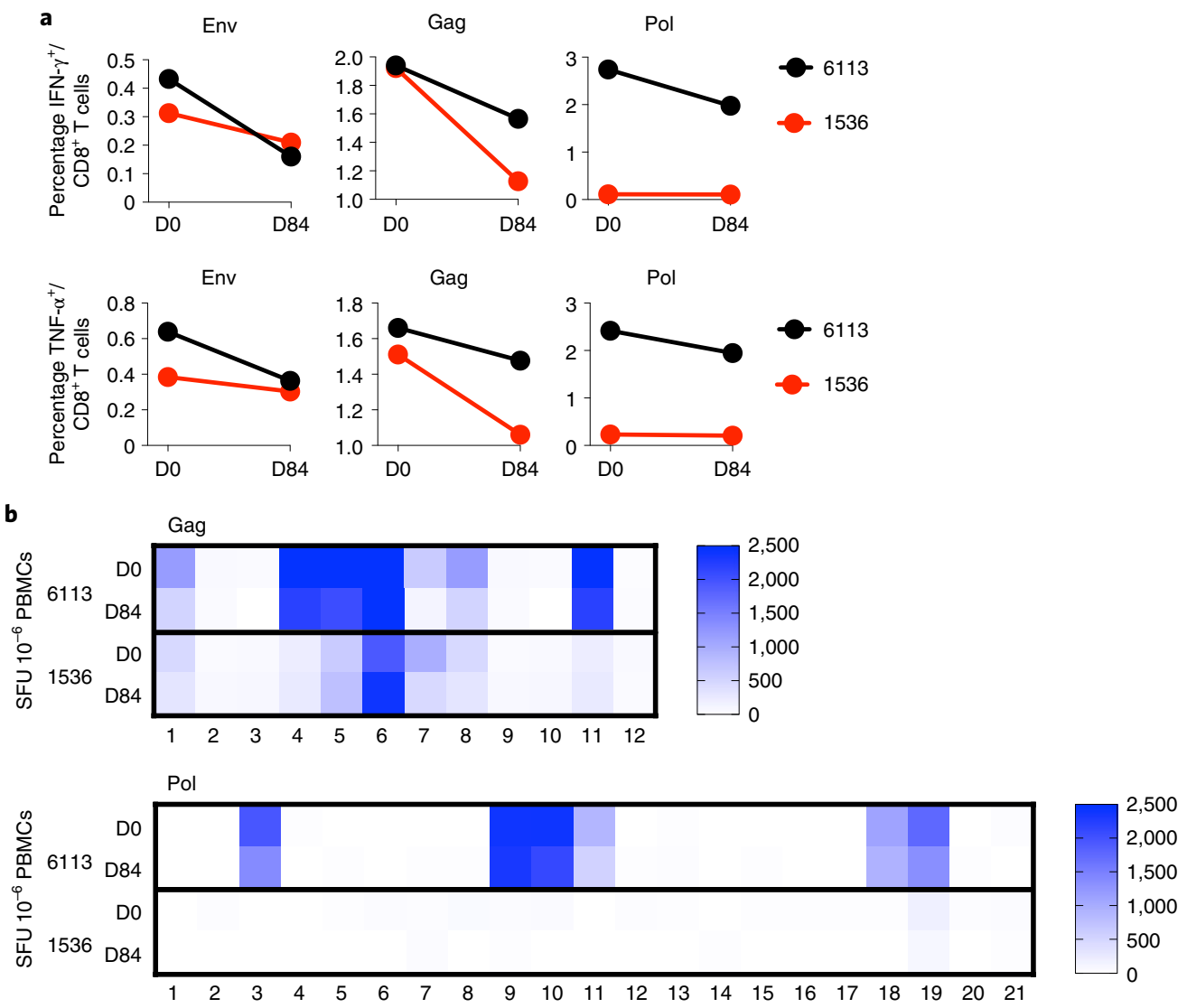

Fig. 4 | Immunologic responses following PGT121 infusion in two long-term suppressors. a, The percentages of Env-, Gag- and Pol-specific CD8 ${ }^{+} T$ cells secreting IFN- $\gamma$ and TNF- $\alpha$ Env are shown for participants 6113 and 1536 at day 0 (D0) and day 84 (D84). b. The magnitude of Gag- and Pol-specific IFN- $\gamma$-secreting cellular immune responses (spot-forming units (SFU) $10^{-6} \mathrm{PBMCs}$ ) is plotted by peptide subpool for participants 6113 and 1536 at D0 and D84.

participants with PGT121 sensitivity and high viral load at baseline had a brisk response, with a median $1.77 \log$ decrease in viral load within a median 8.5 days. Notably, two out of four individuals with a low viral load at baseline demonstrated drug-free viral suppression for $\geq 168$ days following a single infusion of PGT121. For these two participants, viral rebound occurred only when PGT121 was no longer detectable in serum, and their rebound viruses still demonstrated full or partial PGT121 sensitivity. ART-free viral load suppression $\geq 168$ days has not previously been reported in the literature following one dose of an individual or combined bNAb treatment for HIV.

Consistent with previous studies, PGT121 monotherapy in 11 out of $13 \mathrm{HIV}$-infected viremic participants led to the emergence of PGT121 resistance and rebound back to baseline viral loads by day 28 . Among the 12 individuals that displayed at least partial resistance to PGT121 following treatment, resistance could be readily explained in 11 by loss of the glycan at N332 (in seven participants), the loss of the D325 (two participants) or the loss of both N332 and D325 (two participants). In two participants (6292 and 2990), the loss of the D325 form was probably propagated due to recombination ${ }^{25}$ (Extended Data Fig. 5). One participant (6113) had viruses that were sensitive to PGT121 both before and after treatment; this individual maintained drug-free viral suppression through their day 168 visit, and rebound was detected at their next visit on day 252. For this individual it is possible that resistance was never elicited, though it is also possible that resistant viruses might have emerged after day 168 but had already been replaced by a more fit form by the time sampling was performed at day 252 .
The simultaneous emergence of many distinct forms of resistance suggests that these variants may have been present at baseline, either at a low frequency in circulating strains or in the latent reservoir, and selection imposed by the PGT121 antibody rapidly revealed the pre-existing complexity among posttreatment samples. ${ }^{26}$. A hypothesis consistent with these data is that participants may have had a previous antibody response that targeted the V3 epitope region, the virus escaped, the antibody response waned and a more fit PGT121-sensitive form came to dominate the circulating virus quasispecies. These data, together with our observation that PGT121 resistance caused resistance to other bNAbs in only one out of 12 participants, underscore the importance of combining bNAbs that target distinct and nonoverlapping epitopes in future studies for HIV treatment or prevention.

While both PGT121 and 10-1074bind to carbohydrate-dependent epitopes on V3, previous data suggested that PGT121 might have a higher threshold to resistance than 10-1074 due to more promiscuous binding to heterogeneous N-glycan compositions on HIV-1 envelope spikes ${ }^{14,27,28}$. We did not observe such a higher threshold for PGT121 in our study. Three participants (2305, 7190 and 2936) developed complete resistance to both PGT121 and 10-1074 associated with loss of glycan N332, and two participants (2990 and 2319) developed complete resistance to PGT121 but remained highly sensitive to 10-1074 (Extended Data Figs. 5 and 6). These data suggest that there may be additional differences in the binding properties of these two antibodies that could account for varying resistance patterns in rebound viruses.

Of note, our analysis of pathways to resistance following PGT121 administration is limited by the fact that we were able to measure 
only those viruses that were found in the circulation at the specific time point of the blood-draw, and this sample probably does not represent the entirety of viral quasispecies present in the participant. Nevertheless, these are the viruses that dominate in peripheral blood pre- and post-bNAb treatment, driving the infection.

Previous studies have explored whether bNAb treatment for HIV may induce a so-called vaccinal effect, whereby the administration of the bNAb enhances host immune responses and leads to better viral control ${ }^{23,24}$. To investigate this hypothesis, we investigated whether our two participants with long-term suppression had any change in their cellular immune responses. We found that there was no increase in magnitude, phenotype or activation and exhaustion status in either participant following bNAb administration. Our conclusion is that the long-term virologic suppression in these two participants is probably due to the exquisite potency of PGT121, even at levels below the limit of quantitation, coupled with the fact that both participants had low viral loads at baseline. PGT121 may have a higher barrier to resistance than other single bNAbs tested thus far, accounting for this long-term suppression, and/or participants with low viral load at baseline may have a limited number of pre-existing viral quasispecies at the time of PGT121 administration. It is also possible that other, unmeasured, immune responses, such as natural killer cell or complex humoral immune responses, may have exerted some immune-mediated control on viral replication that we were not able to detect.

Finally, our data demonstrate that PGT121 monoclonal antibody was safe and well tolerated in both HIV-uninfected and $\mathrm{HIV}$-infected individuals up to $30 \mathrm{mg} \mathrm{kg}^{-1} \mathrm{IV}$, and at $3 \mathrm{mg} \mathrm{kg}^{-1} \mathrm{SC}$ in HIV-uninfected adults. The half-life of PGT121 was 22 and 14 days in HIV-uninfected and -infected participants, respectively. PGT121 did not appear to generate any functional anti-drug antibodies after a single dose, because no neutralizing anti-PGT121 antibodies were detected at the end of follow-up in any participant.

In summary, PGT121 was safe, well tolerated and had a brisk antiviral effect in viremic, HIV-1-infected participants whose viruses were sensitive to PGT121 at baseline. In two participants with low viral loads at baseline, a single administration of PGT121 was associated with long-term, Ab-mediated viral suppression for up to, and greater than, 6 months. Although the generalizability of our data is limited by our small sample size, they suggest that PGT121 should be tested further for its ability to maintain viral suppression in the setting of ART interruption, or to block HIV acquisition, particularly when used in combination with other bNAbs.

\section{Online content}

Any methods, additional references, Nature Research reporting summaries, source data, extended data, supplementary information, acknowledgements, peer review information; details of author contributions and competing interests; and statements of data and code availability are available at https://doi.org/10.1038/ s41591-021-01509-0.

Received: 3 February 2021; Accepted: 16 August 2021; Published online: 7 October 2021

\section{References}

1. Lynch, R. M. et al. Virologic effects of broadly neutralizing antibody VRC01 administration during chronic HIV-1 infection. Sci. Transl. Med. 7, 319 ra206 (2015).

2. Caskey, M. et al. Antibody 10-1074 suppresses viremia in HIV-1-infected individuals. Nat. Med. 23, 185-191 (2017).

3. Caskey, M. et al. Viraemia suppressed in HIV-1-infected humans by broadly neutralizing antibody 3BNC117. Nature 522, 487-491 (2015).
4. Bar-On, Y. et al. Safety and antiviral activity of combination HIV-1 broadly neutralizing antibodies in viremic individuals. Nat. Med. 24, 1701-1707 (2018).

5. Crowell, T. A. et al. Safety and efficacy of VRC01 broadly neutralising antibodies in adults with acutely treated HIV (RV397): a phase 2, randomised, double-blind, placebo-controlled trial. Lancet HIV 6, e297-e306 (2019).

6. Bar, K. J. et al. Effect of HIV antibody VRC01 on viral rebound after treatment interruption. N. Engl. J. Med. 375, 2037-2050 (2016).

7. Scheid, J. F. et al. HIV-1 antibody 3 BNC117 suppresses viral rebound in humans during treatment interruption. Nature 535, 556-560 (2016).

8. Mendoza, P. et al. Combination therapy with anti-HIV-1 antibodies maintains viral suppression. Nature 561, 479-484 (2018).

9. Walker, L. M. et al. Broad neutralization coverage of HIV by multiple highly potent antibodies. Nature 477, 466-470 (2011).

10. Julien, J.-P. et al. Broadly neutralizing antibody PGT121 allosterically modulates CD4 binding via recognition of the HIV-1 gp120 V3 base and multiple surrounding glycans. PLoS Pathog. 9, e1003342 (2013).

11. Yoon, H. et al. CATNAP: a tool to compile, analyze and tally neutralizing antibody panels. Nucleic Acids Res. 43, W213-W219 (2015).

12. Barouch, D. H. et al. Therapeutic efficacy of potent neutralizing HIV1 -specific monoclonal antibodies in SHIV-infected rhesus monkeys. Nature 503, 224-228 (2013)

13. Borducchi, E. N. et al. Antibody and TLR7 agonist delay viral rebound in SHIV-infected monkeys. Nature 563, 360-364 (2018).

14. Julg, B. et al. Protection against a mixed SHIV challenge by a broadly neutralizing antibody cocktail. Sci. Transl. Med. 9, eaao4235(2017).

15. Moldt, B. et al. Highly potent HIV-specific antibody neutralization in vitro translates into effective protection against mucosal SHIV challenge in vivo. Proc. Natl Acad. Sci. USA 109, 18921-18925 (2012).

16. Jilek, B. L. et al. A quantitative basis for antiretroviral therapy for HIV-1 infection. Nat. Med. 18, 446-451 (2012).

17. Wagh, K. et al. Optimal combinations of broadly neutralizing antibodies for prevention and treatment of HIV-1 clade C infection. PLoS Pathog. 12, e1005520 (2016)

18. Bricault, C. A. et al. HIV-1 neutralizing antibody signatures and application to epitope-targeted vaccine design. Cell Host Microbe 25, e58 (2019).

19. Sok, D. et al. A prominent site of antibody vulnerability on HIV envelope incorporates a motif associated with CCR5 binding and its camouflaging glycans. Immunity 45, 31-45 (2016).

20. Stewart-Jones, G. B. et al. Trimeric HIV-1-Env structures define glycan shields from clades A, B, and G. Cell 165, 813-826 (2016).

21. Rong, L., Dahari, H., Ribeiro, R. M. \& Perelson, A. S. Rapid emergence of protease inhibitor resistance in hepatitis C virus. Sci. Transl. Med. 2, 30ra32 (2010).

22. Rong, L., Feng, Z. \& Perelson, A. S. Emergence of HIV-1 drug resistance during antiretroviral treatment. Bull. Math. Biol. 69, 2027-2060 (2007).

23. Niessl, J. et al. Combination anti-HIV-1 antibody therapy is associated with increased virus-specific T cell immunity. Nat. Med. 26, 222-227 (2020).

24. Nishimura, Y. et al. Early antibody therapy can induce long-lasting immunity to SHIV. Nature 543, 559-563 (2017).

25. Song, H. et al. Tracking HIV-1 recombination to resolve its contribution to HIV-1 evolution in natural infection. Nat. Commun. 9, 1928 (2018).

26. Cohen, Y. Z. et al. Relationship between latent and rebound viruses in a clinical trial of anti-HIV-1 antibody 3BNC117. J. Exp. Med. 215, 2311-2324 (2018).

27. Mouquet, H. et al. Complex-type N-glycan recognition by potent broadly neutralizing HIV antibodies. Proc. Natl Acad. Sci. USA 109, E3268-E3277 (2012)

28. Sok, D. et al. Promiscuous glycan site recognition by antibodies to the high-mannose patch of gp120 broadens neutralization of HIV. Sci. Transl. Med. 6, 236ra263 (2014).

Publisher's note Springer Nature remains neutral with regard to jurisdictional claims in published maps and institutional affiliations.

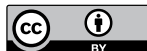

Open Access This article is licensed under a Creative Commons Attribution 4.0 International License, which permits use, sharing, adaptation, distribution and reproduction in any medium or format, as long as you give appropriate credit to the original author(s) and the source, provide a link to the Creative Commons license, and indicate if changes were made. The images or other third party material in this article are included in the article's Creative Commons license, unless indicated otherwise in a credit line to the material. If material is not included in the article's Creative Commons license and your intended use is not permitted by statutory regulation or exceeds the permitted use, you will need to obtain permission directly from the copyright holder. To view a copy of this license, visit http://creativecommons. org/licenses/by/4.0/.

(c) The Author(s) 2021 


\section{Methods}

Study design. The study evaluated the safety, PK and antiviral activity of PGT121 monoclonal antibody (mAb). Part 1 of the study was a single-center, randomized, double-blind, dose-escalation, placebo-controlled trial of PGT121 in HIV-uninfected adults and HIV-infected adults on antiretroviral therapy (ART) at Beth Israel Deaconess Medical Center (BIDMC), Boston, MA, USA. Part 2 of the study was a multicenter, open-label trial of PGT121 in viremic HIV-infected adults not on ART at three sites: BIDMC, Boston, MA; Orlando Immunology Center (OIC), Orlando, FL; and Houston AIDS Research Team (HART), McGovern Medical School at The University of Texas Health Science Center at Houston, TX (all USA). The protocol was approved by the BIDMC Institutional Review Board (IRB), the OIC IRB and the HART Committee for the Protection of Human Subjects. The study was registered at ClinicalTrials.gov (no. NCT02960581). In Part 1 we evaluated three doses (3, 10 and $30 \mathrm{mg} \mathrm{kg}^{-1}$ ) and two routes (IV and SC) of PGT121 given once (Fig. 1a). Each participant in Part 1 received either PGT121 or placebo at a ratio of 4:1 within each subgroup. In Part 2, all participants received a single IV administration of PGT121 at $30 \mathrm{mg} \mathrm{kg}^{-1}$. There was no placebo or blinding for Part 2 .

Study participants. Participants were eligible for the trial across groups if they had a body mass index $>18$ and $<35$ and if they did not have any clinically important acute or chronic medical condition (besides HIV). To enroll, sexually active participants had to be willing to use contraception for three months following investigational product (IP) administration, and could not be pregnant or breastfeeding. Participants were eligible for Group 1 if they were also 18-50years of age, at low risk for HIV infection and willing to maintain low-risk behavior. HIV-infected participants were eligible for either Group 2 or 3 if they were $18-65$ years of age, had $\geq 300 \mathrm{CD} 4$ cells $\mu \mathrm{l}^{-1}$ and no history of acquired immune deficiency syndrome (AIDS)-defining illness within the previous 5 years. HIV-infected participants were eligible for Group 2 if they were currently on ART with suppression of plasma HIV-1 viral load $<50$ copies $\mathrm{ml}^{-1}$ for $>6$ months. HIV-infected participants were eligible for Group 3 if they were not receiving ART, willing to defer ART treatment for at least 56 days after administration of IP (after appropriate counseling) and had an HIV-1 viral load of either 2,000-100,000 or 1002,000 copies $\mathrm{ml}^{-1}$. All participants gave written informed consent and successfully completed an assessment of understanding before the initiation of study procedures.

Randomization and masking. In Part 1, eligible participants were enrolled into the lowest dose subgroup (Group 1A or 2A) depending on their HIV serostatus. Dose subgroups in Groups 1 and 2 were enrolled in parallel. Participants in each subgroup were identified by a unique study identification number and randomized according to the schedule prepared by the Data Coordinating Center (DCC, Emmes Company, LLC) before the start of the study. Participants were automatically assigned a specific allocation number as they were enrolled into the data entry system. An unblinding list (pharmacy list) was provided to the unblinded site pharmacist by the DCC. Study staff and participants were blinded to the allocation of IP. A site pharmacist was unblinded for preparation of the study product. Blinded participants were informed about their assignment (product/placebo) at study completion, once the data were locked. Groups 1 and 2 were unblinded separately after the last participant in the respective groups had completed study participation. Because PGT121 and placebo (saline) appeared identical in the infusion bag, no masking was required. The five participants in each subgroup were randomized at a ratio of four PGT121 recipients to one placebo recipient. At each dose level in Part 1, IP administration was separated by at least $24 \mathrm{~h}$ for each of the first three participants. Randomization ensured that at least two participants received active product and were observed for at least $24 \mathrm{~h}$ before administration to additional participants.

Investigational products. PGT121 is a recombinant, fully human mAb of the IgG1 isotype that binds to the HIV envelope. PGT121 was formulated in a $20 \mathrm{mM}$ acetate, $9 \%$ sucrose, $0.008 \%$ polysorbate $80, \mathrm{pH} 5.2$ formulation buffer at a concentration of $50 \mathrm{mg} \mathrm{ml}^{-1}$. Each 10-ml vial contained $6 \mathrm{ml}$ of PGT121. Placebo was $0.9 \%$ sodium chloride. Participants received either PGT121 or placebo via IV infusion or SC injection, the former over approximately $60 \mathrm{~min}$. No local or topical anesthetic was used before SC injections. IP was injected over a period of 1-5 min, preferably in the abdomen. The maximum volume injected SC per site was approximately $2.5 \mathrm{ml}$. If multiple SC injections were needed, the distance between injection sites was at least 1 inch.

Safety assessments. Local and systemic reactogenicity safety data were collected for 3 days after IP administration (see Supplementary Information for protocol and schedule of procedures). Data on unsolicited AEs were collected until 56 days following IP administration. Potential immune-mediated diseases (pIMDs) were considered AEs of special interest since these could potentially be caused by immune responses to the IP. Data on pIMDs and SAEs were collected through to study Day 168. Blood samples for serum chemistry and hematology were collected at 12 time points throughout the study, as were urine samples for pregnancy testing and urinalysis. Blood samples for HIV-1 viral load and CD4 ${ }^{+}$T-cell count were collected throughout the study from HIV-infected participants. Medical monitoring was provided by a protocol safety review team and an independent safety monitoring committee. Local and systemic AEs were graded by the NIAID
Division of AIDS Table for Grading the Severity of Adult and Pediatric Adverse Events v.2.1, July 2017. For the first $24 \mathrm{~h}$ after IP infusion or injection, any infusion-related reactions, including cytokine release syndrome, were graded by the National Cancer Institute Common Terminology Criteria for Adverse Events v.5.0, 27 November 2017. Peripheral blood was collected to determine PGT121 serum levels, anti-drug antibodies, HIV sequencing and immunogenicity, among other research assessments outlined in the study protocol (Supplementary material).

Antiretroviral therapy counseling. HIV-infected participants who were not on ART received ART counseling upon entering the study and 8 weeks after administration of IP. Participants who had not initiated, or made plans to initiate, ART by the final study visit received ART counseling again at their final study visit. HIV-infected participants who were on ART (Group 2) were counseled on the importance of continuing ART throughout the study, and were not required to interrupt ART after administration of IP.

HIV-1 viral load and CD4 ${ }^{+}$T-cell measurement. Plasma HIV-1 viral load was measured at BIDMC by real-time PCR assay using the Roche COBAS AmpliPrep/ COBAS TaqMan HIV-1 Test, v.2.0 (LLoQ $=23$ copies $\left.\mathrm{ml}^{-1}\right)$, until it was replaced with a transcription-mediated amplification assay using the Hologic Aptima HIV-1 Quant Assay $\left(\mathrm{LLoQ}=32\right.$ copies $\left.\mathrm{ml}^{-1}\right)$. HIV-1 viral load was measured at OIC and HART by real-time PCR using the Abbott Real Time HIV-1 assay (LLoQ $=40$ copies $\left.\mathrm{ml}^{-1}\right)$; assays were performed at LabCorp. CD4 ${ }^{+} \mathrm{T}$-cell counts were measured using a clinical flow cytometry assay performed at either LabCorp or BIDMC.

Determination of PGT121 serum levels. (1) Binding antibody multiplex assay: PGT121 levels were determined on a Bio-Plex instrument (Bio-Rad) using standardized HIV-1 Luminex assays as previously described ${ }^{29-32}$. PGT121 concentrations were representative of three separate assays, where each sample was run in duplicate. The standard curve was PGT121 IgG mAb, titrated in assay diluent. The negative controls were CH58 (an irrelevant mAb) and blank beads. Samples with PGT121 concentrations of LLoQ $<0.5 \mu \mathrm{g} \mathrm{ml}^{-1}$ for HIV-uninfected participants, and $0.71 \mu \mathrm{g} \mathrm{ml}^{-1}$ for HIV-infected participants, at a dilution of 1:50 were designated as 0.5 and 0.71 for plotting purposes for HIV-uninfected and -infected participants, respectively. Samples with PGT121 concentrations above the LLoQ at 1:50 dilution were further tested at various dilution factors to obtain median fluorescent intensity (MFI) in the linear range of the standard curve, and the resulting concentrations from the standard curve were averaged. Samples that were positive at or greater than the limit of detection with an MFI threefold over the preinfusion MFI with a PGT121 concentration lower than LLoQ were designated positive; samples with observed concentrations lower than the LLoQ were designated negative. (2) TZM-bl neutralization assay: the neutralizing actively of passively infused PGT121 was measured as a function of reduction in Tat-regulated luciferase (Luc) reporter gene expression in TZM-bl cells ${ }^{33-36}$. Neutralization titers were measured in pre- and postinfusion immune sera against indicator viruses X2088_c9 and CNE30 (viruses sensitive to neutralization by PGT121) and MuLV (negative control virus). Titers ranged from a minimum of $1: 20$ to a maximum of $1: 1,562,500$, with values outside of this range considered censored. Serum concentrations of PGT121 were calculated for each indicator virus by multiplying the serum $\mathrm{ID}_{50}$ titer at each time point with the median $\mathrm{IC}_{50}$ titer of the PGT121 drug product for each respective virus. The median $\mathrm{IC}_{50}$ titer of PGT121 against viruses X2088_c9 and CNE30 was determined by neutralization assays utilizing the clinical PGT121 drug product (Catalent, no. 1-FIN-2442), tested at a primary concentration of $10 \mu \mathrm{g} \mathrm{ml}^{-1}$ with fivefold dilution series. Replicate assays were performed on multiple days by independent operators ( $n=12$ for each virus). All preinfusion serum samples from HIV-infected or -uninfected participants were shown to have no neutralizing activity against PGT121-specific indicator viruses X2088_c9 and CNE30. For Group 2 participants (HIV-infected on ART), serum samples were tested using viruses CNE30, X2088_c9 and MuLV pseudotyped using an SG3Env backbone vector engineered with three site-directed mutations to confer resistance to inhibition by antiretroviral drugs present in the serum samples (triple mutant no. Q148H/K101P/Y181C).

\section{Measurement of ADA levels. Anti-drug antibodies against PGT121} were measured using a qualified electrochemiluminescence (ECL)-based bridging immunoassay. In brief, test serum samples were first subjected to an acid-dissociation step in glycine- $\mathrm{HCl}$ acid buffer on a plate shaker for $1 \mathrm{~h}$. Acid-treated samples were mixed (1:1) with a mastermix containing equimolar amounts $\left(0.5 \mu \mathrm{g} \mathrm{ml}^{-1}\right)$ of PGT121 covalently conjugated to biotin or ruthenium labels and incubation on a plate shaker overnight. After overnight incubation, samples were transferred to a 96-well functionalized streptavidin plate (Meso Scale Discovery) and shaken for $1 \mathrm{~h}$ on a plate shaker. The streptavidin plate was washed several times, Tris-based read buffer containing tripropylamine (TPA) was added and the presence of ADA bridging complexes detected on a MESO QuickPlex SQ-120 plate reader (Meso Scale Discovery). The ECL signal generated was proportional to the level of ADA bridging complexes captured in each sample. Any samples that were above the assay floating cutoff point for positivity were considered putative ADA positive and were reanalyzed in a competitive inhibition confirmatory assay using the same assay format in the presence and absence of a molar excess $\left(200 \mathrm{\mu g} \mathrm{ml}^{-1}\right)$ of unlabeled PGT121 in the assay mastermix. 
Inhibition of $>20 \%$ in the presence of excess PGT121 was used to confirm ADA positivity. Functional anti-PGT121 ADA activity was measured in TZM.bl cells as previously described ${ }^{37}$. Briefly, patient serum samples were serially diluted in the presence of a partial inhibitory dose of PGT121, and neutralizing activity against the PGT121-sensitive virus X2088_c9 was measured. ADA titers are expressed as the reciprocal sample dilution required to decrease the neutralizing activity of the bNAb by $50 \%$. A murine anti-PGT121 antibody was utilized as a positive control.

Sequencing of participant HIV-1 env genes and production of pseudoviruses. Single-genome amplification (SGA) assays were performed essentially as previously described ${ }^{38}$. Briefly, HIV-1 RNA was isolated and reverse transcribed to viral complementary DNA. First-round PCR was carried out with Q5 High-Fidelity 2X Master Mix (NEB) together with HIV B primers. Amplicons from cDNA dilutions showing $<30 \%$ positive were considered to have resulted from a single cDNA amplification and were processed for sequencing. For each sample, 15-30 sequences were analyzed. Selected viral sequences isolated from the plasma of each participant by SGA were used to generate pseudoviruses as previously described ${ }^{34}$.

Assessment of ART drug levels in plasma. Plasma samples from 6113 and 1536 from visit 11 were quantitatively analyzed for emtricitabine and lamivudine at the University of Nebraska Medical Center. These two antiretroviral drugs were chosen for testing because at least one of them is present in commonly used combination antiretroviral regimens.

Immunogenicity assessments. HIV-specific T-cell responses were measured by IFN- $\gamma$ enzyme-linked immunospot (ELISPOT) assay using potential T-cell epitopes (PTE) Env, Pol and Gag peptide libraries ${ }^{39,40}$. ELISPOT assay was performed as follows. White membrane plates (Millipore) were coated at $4{ }^{\circ} \mathrm{C}$ overnight with $10 \mu \mathrm{g} \mathrm{ml}^{-1}$ anti-human IFN- $\gamma$ (Mabtech). Rested peripheral blood mononuclear cells (PBMCs) were plated at $2 \times 10^{6}$ with PTE Env, Pol or Gag pools at $2 \mu \mathrm{g} \mathrm{ml}^{-1}$ for $18 \mathrm{~h}$ at $37^{\circ} \mathrm{C}$. Development was achieved by the addition of biotin (Mabtech), antibiotin (VectorLabs) and chromagen (Pierce). Background subtraction was noted by matched DMSO peptide concentration of $0.4 \%$. Multiparameter intracellular cytokine staining (ICS) assays were performed essentially as described ${ }^{41}$. ICS assays were performed with $10^{6} \mathrm{PBMCs}$ incubated for $6 \mathrm{~h}$ at $37^{\circ} \mathrm{C}$ with medium, $10 \mathrm{pg} \mathrm{ml}^{-1}$ phorbol myristate acetate and $1 \mu \mathrm{g} \mathrm{ml}^{-1}$ ionomycin (Sigma-Aldrich), or $1 \mu \mathrm{g} \mathrm{ml}{ }^{-1}$ HIV-1 Env, Gag or Pol peptide pools. Cultures contained monensin (GolgiStop, BD Biosciences), brefeldin A (GolgiPlug, BD Biosciences) and $1 \mu \mathrm{g} \mathrm{ml}^{-1} \mathrm{mAb}$ against human CD49d (clone 9F10). Cells were then stained with predetermined titers of mAbs against CD3 (clone SP34.2, Alexa 700), CD4 (clone L200, BV786), CD8 (clone SK1, APC H7), CD38 (clone HIT2, BUV805) and PD-1 (clone EH12.2H7, Pacific Blue), and stained intracellularly with IFN- $\gamma$ (clone B27, BUV395), IL-2 (clone MQ1-17H12, BUV737), TNF- $\alpha$ (clone Mab11, BV650) and Ki67 (clone B56, FITC). IFN- $\gamma$ backgrounds were $<0.05 \%$ in PBMCs.

Endpoints. The primary endpoints were, for safety and tolerability: (1) proportion of participants with moderate or greater reactogenicity (for example, solicited AEs) for 3 days following administration of PGT121 mAb; (2) proportion of participants with moderate or greater and/or PGT121 mAb-related unsolicited AEs, including safety laboratory parameters, following administration of PGT121 mAb for the first 56 days following administration of IP; and (3) proportion of participants with PGT121 mAb-related SAEs throughout the study period. The primary endpoints for PK were elimination half-life $\left(t_{1 / 2}\right)$, clearance $(\mathrm{CL} / \mathrm{F})$, volume of distribution $(\mathrm{Vz} / \mathrm{F})$, area under the concentration decay curve (AUC) and impact of viral load and/or ART on PGT121 disposition (elimination $t_{1 / 2}$ ), clearance $(\mathrm{CL} / \mathrm{F})$, volume of distribution $(\mathrm{Vz} / \mathrm{F})$ and total exposure. The primary endpoint for antiviral activity among viremic HIV-infected adults not on ART was a change in plasma HIV-1 RNA levels from baseline (mean of pre-entry and entry values). The secondary endpoints were change in serum anti-PGT121 antibody titers from baseline, change in $\mathrm{CD} 4^{+} \mathrm{T}$-cell count and frequency compared to baseline as measured by single-platform flow cytometry, and development of HIV-1 sequence variations in epitopes known to result in reduced PGT121 mAb neutralization susceptibility. Additional exploratory assessments could include, but were not limited to, the following: HIV-specific IgG/IgA binding responses by ELISA; HIV-specific cellular immune responses by ELISPOT; HIV-specific antibody function by ADCC, ADCP and ADCVI assays; resistance mutations to current ARVs; PGT121 mAb levels in mucosal secretions; changes in total HIV-1 DNA and two-long terminal repeat (LTR) circular HIV-1 DNA in resting or total CD4 ${ }^{+}$T cells; and in vitro neutralization of HIV isolates with participant's serum following administration of PGT121 mAb. Available samples from time points during the optional long-term extension (LTE) phase were also used for determination of long-term durability of immune responses. The primary endpoints for safety, tolerability and PK were changed in protocol v.6.0, to include evaluation of both IV and SC routes, after the addition of subgroup $1 \mathrm{D}$ to the trial design. All primary endpoints were also changed in protocol v.8.0, to include evaluation of data from participants enrolled in the optional LTE phase that allowed additional follow-up of participants beyond day 168 (see Supplementary material for all protocol versions).

Sample size and statistical analyses. Because this was an exploratory proof-of-concept trial, analyses are descriptive and sample sizes were selected based on collection of key information necessary to plan future trials. (1) The sample size for safety and tolerability analysis was 35-56 participants, according to the dose-escalation design. For life-threatening AEs related to IP: if none of the 12 (maximum 18) participants receiving IP experienced such reactions, the 95\% upper confidence bound for the rate of these AEs in the population was calculated to be $26.5 \%$ (or $18.5 \%$ when $n=18$ ). The frequency of moderate or greater reactogenicity events was determined and compared between groups. The frequency of SAEs adjudged possibly, probably or related to the IP was determined. All AEs were analyzed and grouped by seriousness, severity and relationship to the IP (as judged by the investigator). An interim analysis of group data was carried out according to the study schema without unblinding the study to investigators or participants. At the end of the study, a full analysis was prepared. Unused and spurious data were listed separately and excluded from the statistical analysis. Missing data were excluded from the statistical analysis. (2) The sample size for PK analysis was four PGT121 and one placebo recipient, sufficient to provide information about the PK of PGT121. The data were fit to standard two-compartment population models using the stochastic approximation expectation-maximization estimation method in Monolix (v.2019R1, Lixoft SAS, 2019). Residual variability was estimated using an additive plus proportional error model, and models were performed separately by HIV infection status. Correlations between confidence interval $(\mathrm{Cl})$ and volume of the central compartment $(\mathrm{Vc})$ were significant (Pearson's correlation coefficient $P \leq 0.05)$ and were included in the models. Median-adjusted, $\log _{10}$-transformed weight was significantly correlated (Pearson's correlation coefficient $P \leq 0.05$ ) with the volume of the peripheral compartment $(\mathrm{Vp})$ in the HIV-uninfected model and with $\mathrm{Vc}$ and $\mathrm{Cl}$ in the HIV-infected model, and those relationships were also included in the models. Distribution and elimination half-lives were computed using the resulting model. AUC was estimated by calculating the integral of the predicted concentration time curve from infusion to infinity. Peak concentration $\left(C_{\max }\right)$ was computed as the maximum observed concentration. Summary descriptive results of individual estimates of PK parameters, including AUC, $C_{\max }, t_{1 / 2}$ and clearance results, are reported by dose cohort. Correlation between PK and reported safety and pharmacodynamic outcomes also explored parameters to examine exposure-effect relationships. (3) The frequency and levels of anti-PGT121 antibodies were calculated and tabulated. (4) The sample size for virologic analysis in Group 3A was nine participants. The primary outcome for this analysis was defined as change in $\log _{10}$ viral load between Day 0 (day of infusion) and Day 7. No placebo participants were enrolled as part of this design. For the analysis of sample size and power, $\log _{10}$ viral load differences from baseline for each participant were simulated from a normal distribution, with a standard deviation of 0.5 . This value was chosen by examining a study of the antiretroviral drug raltegravir, which demonstrated a mean estimated standard deviation of the change in baseline of 0.47 (ref. ${ }^{42}$ ). This is a conservative estimate, as the variability of viral loads near the lower range might be expected also to be lower. The statistical test performed was the signed-rank test, which incorporated the 'shift' parameter of $-0.9 \log _{10}$. An evaluation of potential harm (increased viral load) was also performed with the signed-rank test, which examined the null hypothesis of no change in viral load (a shift of $0 \log _{10}$ following IP administration) against the one-sided alternative hypothesis that viral load is increased following IP administration. Each efficacy test was performed at the level $\alpha=0.05$, and each test for harm was performed at the level $2 \alpha=0.10$, to provide additional sensitivity in detection of potential harm. (5) The sample size for antiviral activity in Group 3D was up to six participants. No efficacy endpoints were tested in Group 3D because participants were HIV infected with low viral loads at baseline $\left(102-2 \times 103\right.$ copies $\left.\mathrm{ml}^{-1}\right)$. Immunologic and virologic endpoints were determined as described in protocol v.8.0 section 4.1 (Supplementary material).

Viral neutralization sensitivity modeling. As shown previously, in vitro neutralization curves can be accurately modeled as Hill curves, using $\mathrm{IC}_{50}$ and $\mathrm{IC}_{80}$ titers to fix the half-maximum and slope parameters ${ }^{17}$. When both $\mathrm{IC}_{50}$ and $\mathrm{IC}_{80}$ were within the concentrations tested, this procedure was used to calculate the percentage neutralization of a virus at a given PGT121 concentration. However, when $\mathrm{IC}_{50}$ or $\mathrm{IC}_{80}$ titers were outside the concentration ranges tested, we fit a Hill curve to the raw percentage neutralization versus concentration data. Curve fitting was done using absolute error minimization, utilizing the L-BFGS-B algorithm as implemented in the Python Scipy Optimize package: https://docs.scipy.org/doc/ scipy/reference/optimize.minimize-lbfgsb.html. The instantaneous inhibitory potential (IIP) was calculated as $-\log _{10}(1.0-f)$, where $f$ is the fraction neutralized at a given bNAb concentration ${ }^{16}$. Average IIP for baseline viruses in Extended Data Fig. 4 was calculated as follows. First, for each participant, bNAb concentrations were extracted for 1 day following infusion up to the time point of minimum viral load. The former was to account for distribution of bNAb to tissues. Next, for each of these concentrations, IIP was calculated as above for each baseline virus for that participant, and the time-averaged IIP found by numerically integrating the IIP curve as a function of time, divided by integral of unity for the same time points. Simpson's rule, as implemented in Scipy, was used for calculations (https:// docs.scipy.org/doc/scipy/reference/generated/scipy.integrate.simpson.html). These time-averaged IIP values for each baseline virus from each participant were averaged to determine the average IIP for each participant. The same procedure was followed to determine the average rebound virus IIP, as shown in Extended 
Data Fig. 3, with the exception of using PGT121 concentrations from the time of minimum viral load to that to rebound.

Viral load decline and rebound characteristics. We used three characteristics of viral load trajectories in each participant to capture the antiviral efficacy of PGT121: (1) maximum viral load drop from baseline, (2) initial decline rate of viral load following infusion and (3) time to viral rebound. The calculation of the first characteristic was straightforward for all participants, except for four participants for whom the minimum viral load was below the lower limit of quantitation $\left(\mathrm{LLoQ}=40\right.$ copies $\mathrm{ml}^{-1}$ ); for such participants the minimum viral load was assumed to be 40 copies $\mathrm{ml}^{-1}$. For the second characteristic, we calculated the average slope of $\log _{10}$ viral load over time using the following strategy. For each participant, we first identified the time window of consistent viral load decline as that between minimum viral load and the earliest time point following infusion such that viral load consistently fell over time in this window. This strategy ensured that the early nonmonotonic behavior of viral load (as seen for participants 1536, 4236 and so on) would not impact calculation of decline rate. In case of multiple time points with minimum viral load, the earliest was chosen. For all time points in the window of viral load decline, the rate was calculated as the slope of $\log _{10}$ viral load versus time curve. Slopes were calculated using a robust linear regression algorithm, the Theil-Sen estimator as implemented in Scipy (https://docs.scipy. org/doc/scipy-0.15.1/reference/generated/scipy.stats.mstats.theilslopes.html), and time points with viral load below LLoQ were ignored. For two participants, 1536 and 4236 , decline rates could not be determined due to the combination of highly nonlinear early viral load kinetics and the minimum viral load dropping below LLoQ; these participants are excluded from the data in Extended Data Fig. 3c,d. For two participants, 9372 and 5257, because viral loads did not decline sufficiently due to PGT121 resistance at baseline, the average slope of viral load for the first 10 days was used. The third characteristic was the time to rebound, for which we assumed that rebound or restoration of viremia is characterized by viral loads $0.5 \log _{10}$ below baseline preinfusion viremia, or higher. Thus, the time to rebound was calculated as that between infusion and the earliest time point that met the rebound viral load criterion.

Sequence analysis. Phylogenetic trees were constructed using IQ-tree ${ }^{43}$, and recombination analysis was performed with $\mathrm{RAPR}^{25}$, both via interfaces at the Los Alamos Database. Sequences are available at GenBank (see Supplementary Table 12 for accession numbers).

Important changes to methods after trial commencement. Eligibility criteria were changed in protocol v.5.0 to remove the inclusion criteria of CD4 nadir $>200$, and to expand the age range for Group 2 and 3 participants, from 18-50 to $18-65$ years of age. These changes were made to facilitate recruitment and to meet the approval of the safety monitoring committee (SMC), protocol safety review team (PSRT) and the BIDMC IRB. Protocol v.6.0 added a subcutaneous administration arm to Part 1. The study design was changed in protocol v.7.0 to eliminate subgroups 3B, 3C, 3E and 3F, which would have evaluated lower doses of PGT121 in viremic individuals but were not feasible to enroll. A LTE was added in protocol v.8.0 to allow additional follow-up of participants beyond day 168 . Protocol v.6.0, 7.0 and 8.0 were also approved by the SMC, PSRT and BIDMC IRB.

Reporting Summary. Further information on research design is available in the Nature Research Reporting Summary linked to this article.

\section{Data availability}

All viral sequences identified in this study are publicly available via GenBank (see Supplementary Table 12 for GenBank accession numbers). Comprehensive data on HIV genetic sequences and immunological epitopes used for analysis in this study are publicly available via Los Alamos National Laboratory (hiv.lanl.gov/ content/index, with most relevant sequences included in Supplementary material). Additional requests for access to the study data can be submitted to D.H.B. (dbarouch@bidmc.harvard.edu). Data containing protected health information or that may identify a participant are restricted, and therefore additional data requests must be reviewed before release.

\section{References}

29. Haynes, B. F. et al. Immune-correlates analysis of an HIV-1 vaccine efficacy trial. N. Engl. J. Med. 366, 1275-1286 (2012).

30. Tomaras, G. D. et al. Initial B-cell responses to transmitted human immunodeficiency virus type 1: virion-binding immunoglobulin $\mathrm{M}(\operatorname{IgM})$ and IgG antibodies followed by plasma anti-gp41 antibodies with ineffective control of initial viremia. J. Virol. 82, 12449-12463 (2008).

31. Tomaras, G. D. et al. Vaccine-induced plasma IgA specific for the $\mathrm{C} 1$ region of the HIV-1 envelope blocks binding and effector function of IgG. Proc. Natl Acad. Sci. USA 110, 9019-9024 (2013).

32. Yates, N. L. et al. HIV-1 gp41 envelope IgA is frequently elicited after transmission but has an initial short response half-life. Mucosal Immunol. 6, 692-703 (2013).
33. Montefiori, D. C. Evaluating neutralizing antibodies against HIV, SIV, and SHIV in luciferase reporter gene assays. Curr. Protoc. Immunol. 12, 12.11 (2005).

34. Seaman, M. S. et al. Tiered categorization of a diverse panel of HIV-1 Env pseudoviruses for assessment of neutralizing antibodies. J. Virol. 84, 1439-1452 (2010).

35. Li, M. et al. Genetic and neutralization properties of subtype $\mathrm{C}$ human immunodeficiency virus type 1 molecular env clones from acute and early heterosexually acquired infections in Southern Africa. J. Virol. 80, 11776-11790 (2006).

36. Li, M. et al. Human immunodeficiency virus type 1 env clones from acute and early subtype B infections for standardized assessments of vaccine-elicited neutralizing antibodies. J. Virol. 79, 10108-10125 (2005).

37. Seaman, M. S. et al. Optimization and qualification of a functional anti-drug antibody assay for HIV-1 bnAbs. J. Immunol. Methods 479, 112736 (2020).

38. Keele, B. F. et al. Identification and characterization of transmitted and early founder virus envelopes in primary HIV-1 infection. Proc. Natl Acad. Sci. USA 105, 7552-7557 (2008).

39. Barouch, D. H. et al. Vaccine protection against acquisition of neutralizationresistant SIV challenges in rhesus monkeys. Nature 482, 89-93 (2012).

40. Li, F. et al. Peptide selection for human immunodeficiency virus type 1 CTL-based vaccine evaluation. Vaccine 24, 6893-6904 (2006).

41. Borducchi, E. N. et al. Ad26/MVA therapeutic vaccination with TLR7 stimulation in SIV-infected rhesus monkeys. Nature 540, 284-287 (2016).

42. Andrade, A. et al. Three distinct phases of HIV-1 RNA decay in treatment-naive patients receiving raltegravir-based antiretroviral therapy: ACTG A5248. J. Infect. Dis. 208, 884-891 (2013).

43. Trifinopoulos, J., Nguyen, L. T., von Haeseler, A. \& Minh, B. Q. W-IQ-TREE: a fast online phylogenetic tool for maximum likelihood analysis. Nucleic Acids Res. 44, W232-W235 (2016)

\section{Acknowledgements}

We thank the participants and staff at the Center for Virology and Vaccine Research Clinical Trials Unit, the Harvard Catalyst Clinical Research Center, Orlando Immunology Clinic and Houston AIDS Research Team. This project was supported by the Bill and Melinda Gates Foundation Collaboration for AIDS Vaccine Discovery (CAVD; grant no. OPP1107669) and National Institutes of Health (NIH; nos AI149670, AI145801, AI129797, AI128751, AI126603, AI124377 and OD024917 to D.H.B.; AI106408 to B.J.; TR001102 (Harvard Catalyst); and AI114381 to K.E.S.). M.S.S. was supported by CAVD grant no. OPP1146996. Portions of this work were performed under the auspices of the US Department of Energy and supported by NIH grant nos. AI028433, OD011095 and AI131365 (to A.S.P.). We thank A. Podany, College of Pharmacy, University of Nebraska Medical Center, for performing emtricitabine and lamivudine quantification assays. We thank the following members of the International AIDS Vaccine Initiative: A. Lombardo, H. Park, E. Sayeed, K. Syvertsen, J. Ackland, M. Schroeter, V. Sharma, J. Hare, M. Fong Lim, N. Williams and K. Crisafi. BIDMC thanks S. Walsh, A. Hale, R. Fogel and T. Makoni for their contributions. The funding source was the Bill \& Melinda Gates Foundation. The funders were involved in the study design, study operations, data collection, data analysis, data interpretations and reviewing and editing the manuscript. The PGT121 program leads (D.H.B., K.E.S. and B.J.) and data and statistical lead (A.D.) had access to all data. The PGT121 program leads had final responsibility for the decision to submit for publication.

\section{Author contributions}

K.E.S., B.J. and D.H.B. designed and led the study. K.E.S. and B.J. were protocol cochairs. K.E.S., R.C.A. and E.D. were site principal investigators. C.S.T., R.Z., S.R.W., C.-P.R., A.N.M. and S.L. were coinvestigators. J.L.A. and D.G.K. were clinical staff. M.S.S., L.F.M., J.N., E.N.B., P.A., J.L., L.P., A.C., R.N., Z.L., A. Setaro, J.S. and Z.C. performed virologic and immunologic assays. H.C.G., L.S. and F.H.P. represented the sponsor, IAVI. T.C., A.S.P., K.W., E.E.G. and B.K. performed analysis of HIV-1 viral sequences. C.B., A. Sato, B.M. and A.C. performed statistical analysis of primary endpoints. N.L.Y. and G.D.T. performed PK analysis. All authors contributed to the writing and editing of the report and approved the final version.

\section{Competing interests}

The authors declare no competing interests.

\section{Additional information}

Extended data is available for this paper at https://doi.org/10.1038/s41591-021-01509-0.

Supplementary information The online version contains supplementary material available at https://doi.org/10.1038/s41591-021-01509-0.

Correspondence and requests for materials should be addressed to Dan H. Barouch. Peer review information Nature Medicine thanks Marina Caskey, Victor Volovici and the other, anonymous, reviewer(s) for their contribution to the peer review of this work. Alison Farrell is the primary editor on this article and managed its editorial process and peer review in collaboration with the rest of the editorial team.

Reprints and permissions information is available at www.nature.com/reprints. 
a

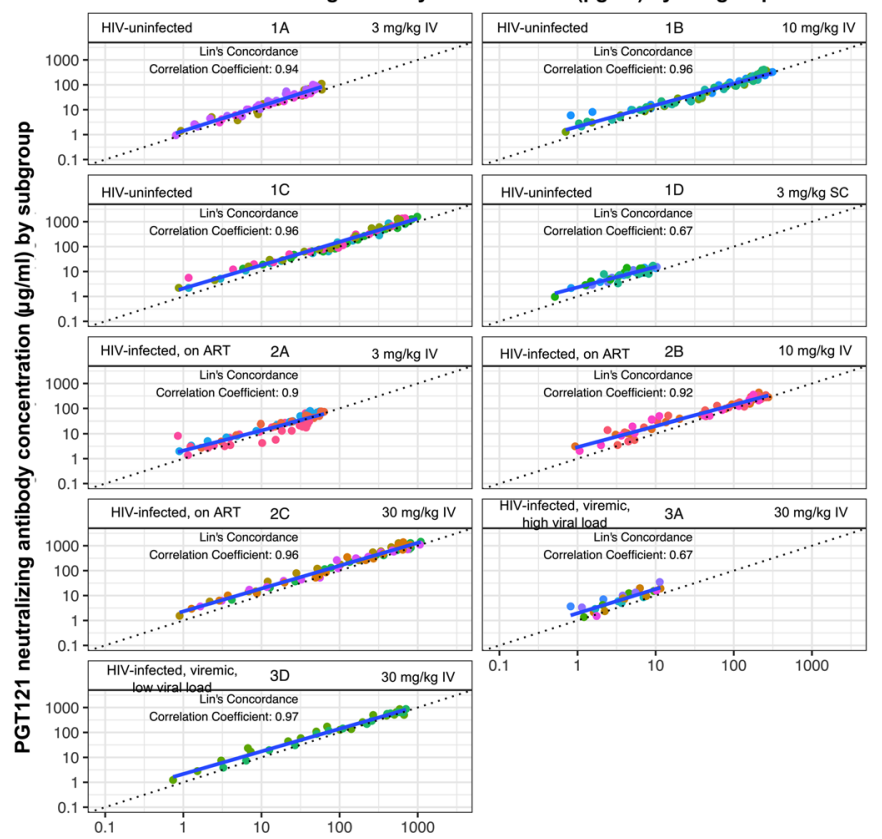

b

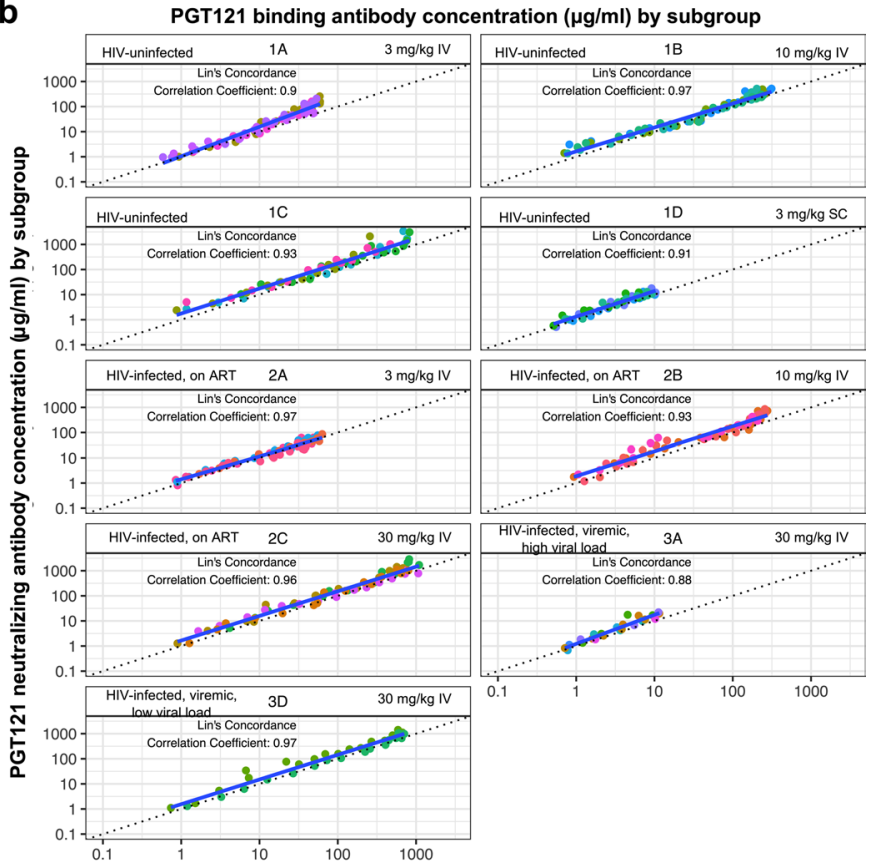

Extended Data Fig. 1 | Correlation between PGT121 neutralizing and binding antibody concentrations. Concordance between PGT121 concentrations measured by binding and neutralizing antibody assays using pseudovirus strains CNE30 (a) and X2088_c9 (b). The dotted line is the identity line. Solid line is the trend line. Data are colored by participant. Lin's concordance correlation coefficient is a measurement of accuracy and precision which is used to assess agreement between two sets of measurements. 


\section{a}
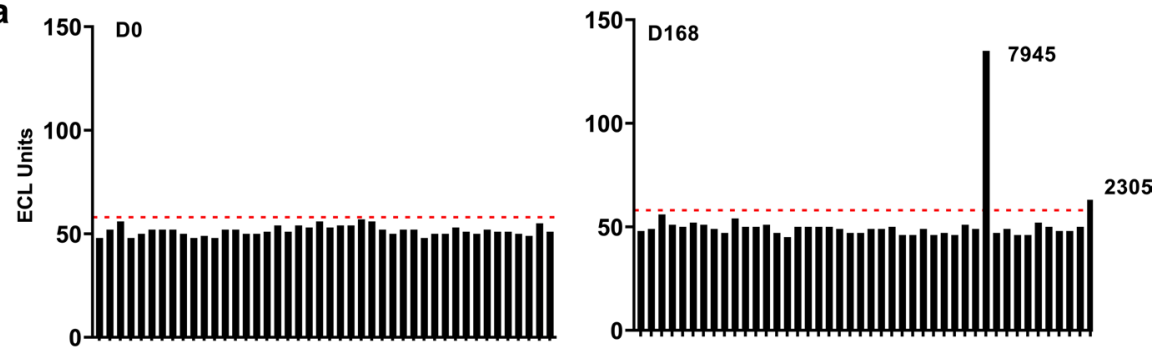

b

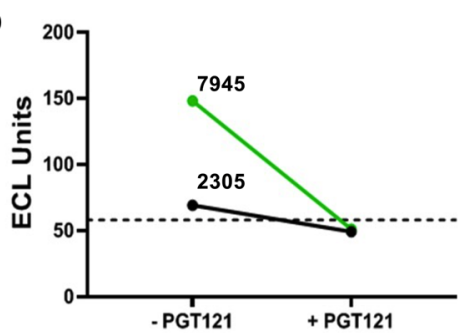

C

\begin{tabular}{|l|l|l|}
\hline PTID & Day & ADA ID50 Titer \\
\hline 7945 & 0 & $<20$ \\
\cline { 2 - 3 } & 168 & $<20$ \\
\hline 2305 & 0 & $<20$ \\
\cline { 2 - 3 } & 168 & $<20$ \\
\hline
\end{tabular}

Extended Data Fig. 2 | Anti-drug antibody responses. (a) Binding anti-drug antibodies at day 0 and day 168 by participant (each black bar represents a separate participant). The red dotted line indicates the run-specific floating cut-point. (b) Binding anti-drug antibodies in the setting of PGT121 or no PGT121 competition in participants 7945 and 2305. (c) Anti-PGT121 neutralizing activity (ID50 titers) at days 0 and 168 for participants 7945 and 2305. 
Full Sensitivity at Baseline $(\mathrm{N}=8)$

High Viral Load $(\mathrm{N}=4)$
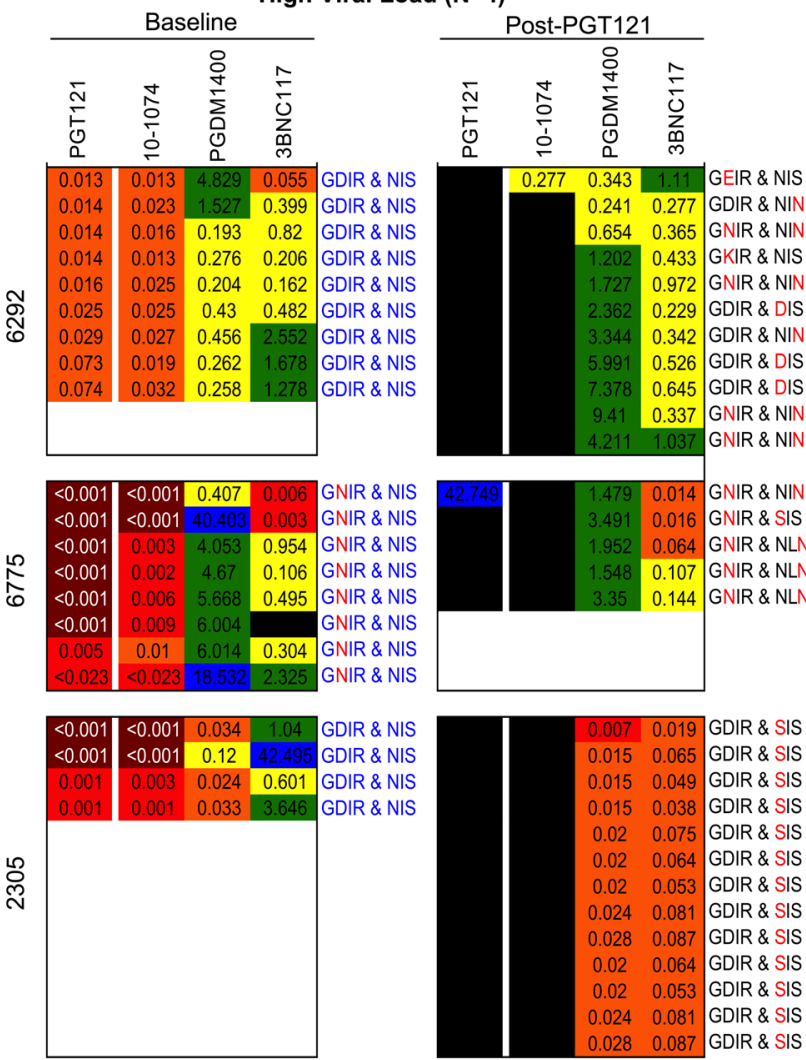

GDIR \& NIS GDIR \& NIS 1 GDIR \& NIS GDIR \& NIS
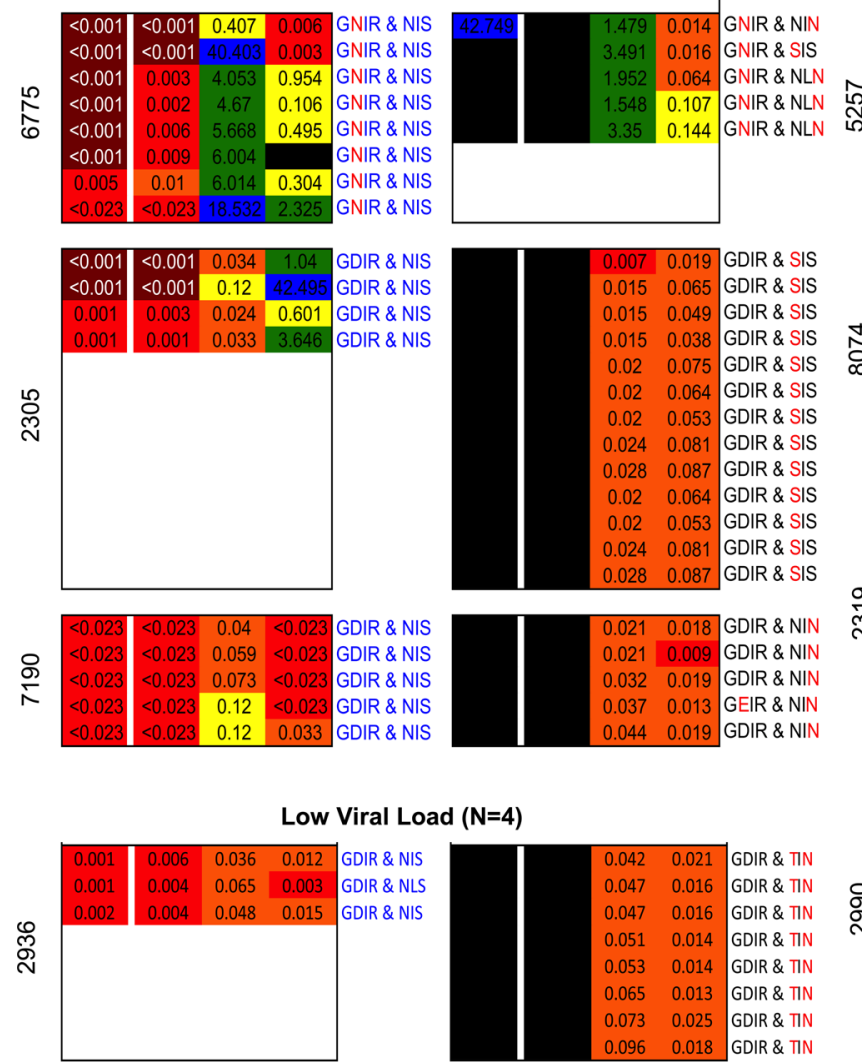

\begin{tabular}{l|l|l|l|l|l|}
\hline 0 & 0.003 & 0.003 & 0.002 & 0.031 & GDIR \& NIT \\
\hline
\end{tabular}

$\stackrel{\mathscr{m}}{\text { พ }}$
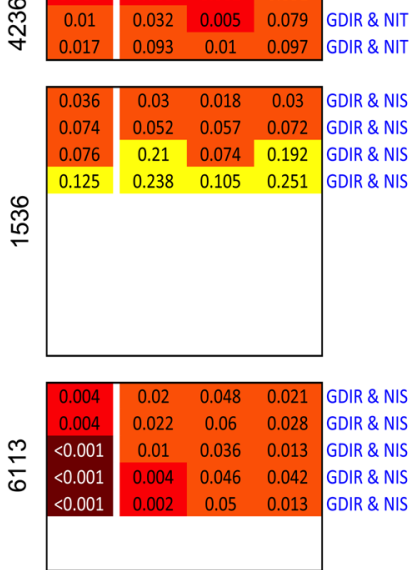
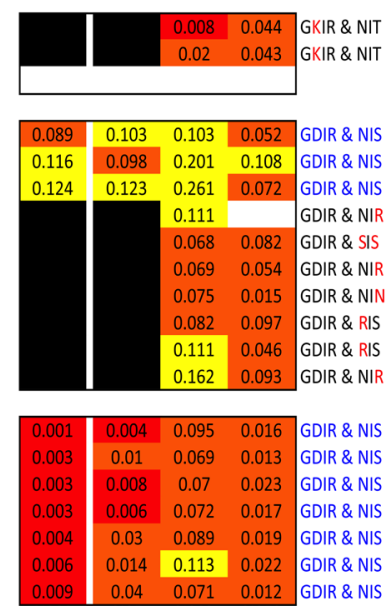
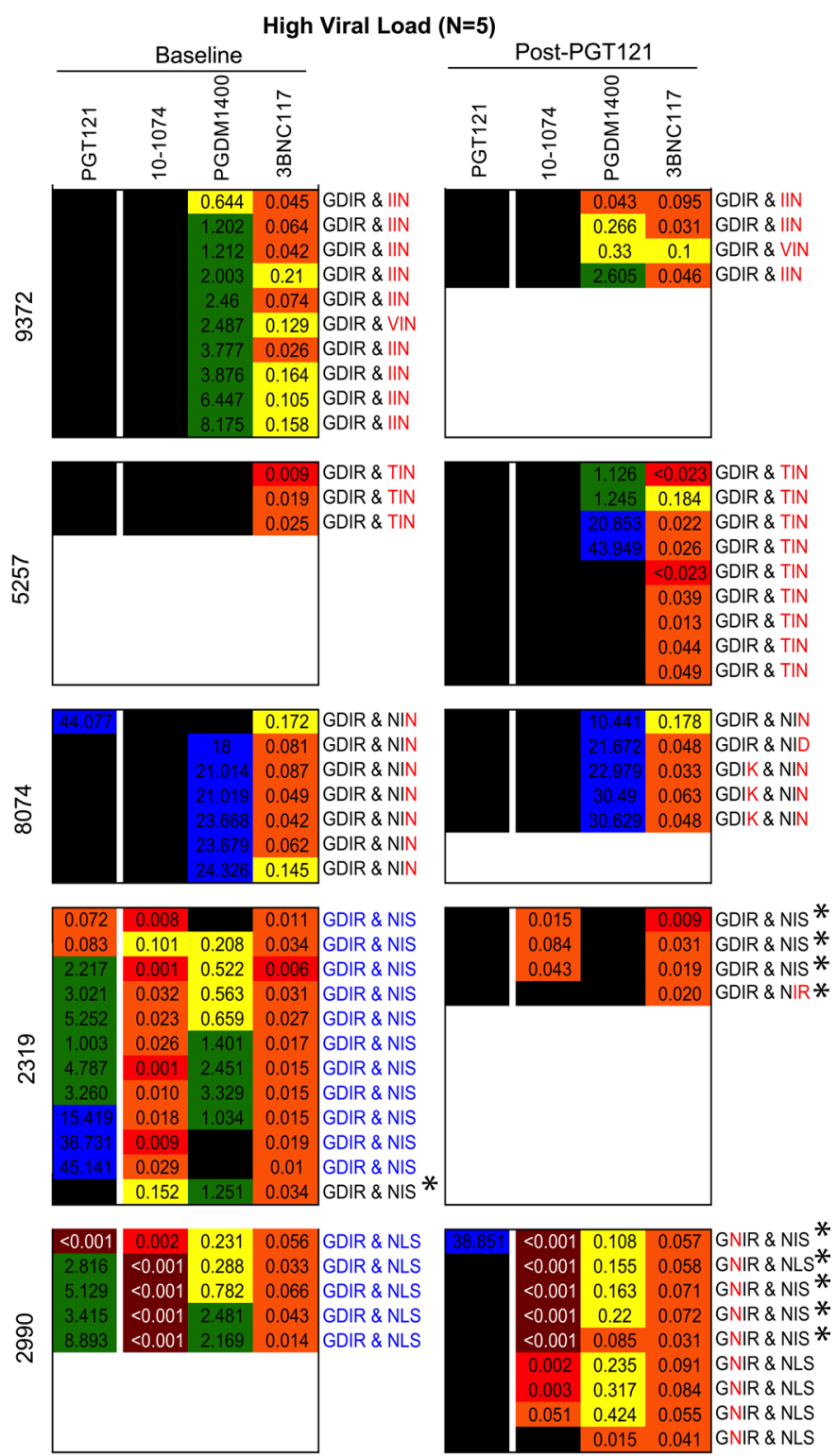

\section{IC50}

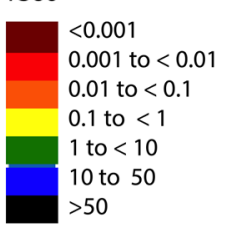

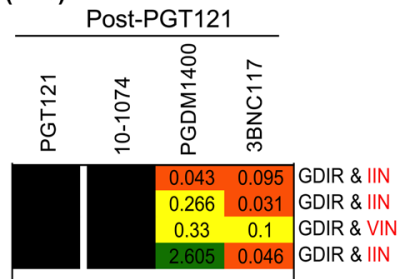


* Glycan at N413

Extended Data Fig. 3 | See next page for caption. 


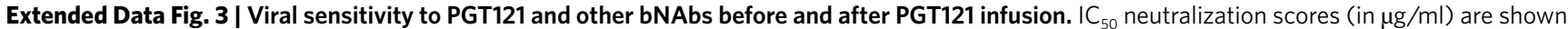
for each participant: PGT121 is shown on the left, followed by 10-1074 that also targets the V3 glycan, then a V2 apex bNAb (PGDM1400), then a CD4bs bNAb (3BNC117). Baseline and post PGT121 treatment values are shown, followed by the amino acid sequence of the GDIR motif (HXB2 positions 324-327) and the N-linked glycosylation motif (positions 332-334). Blue amino acids indicate sensitivity signatures for PGT121, while black and red amino acids indicate resistance signatures. 
A

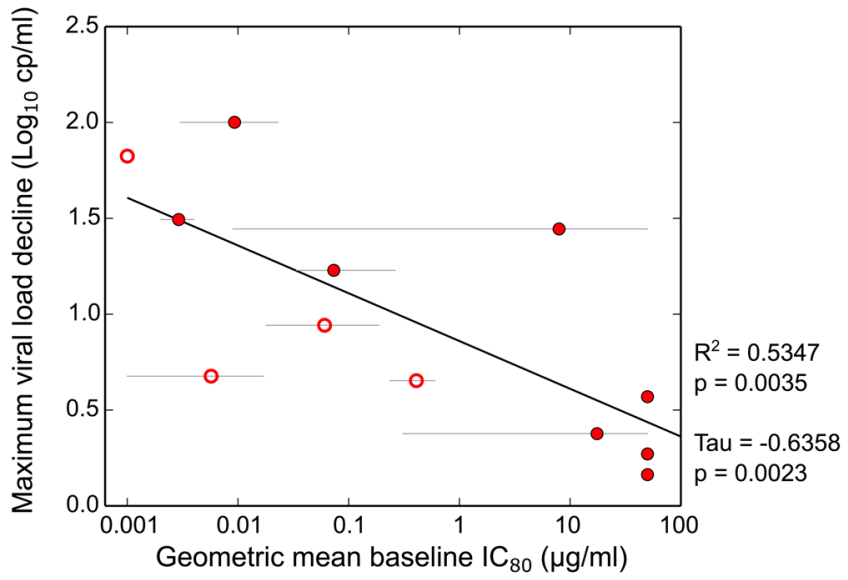

C

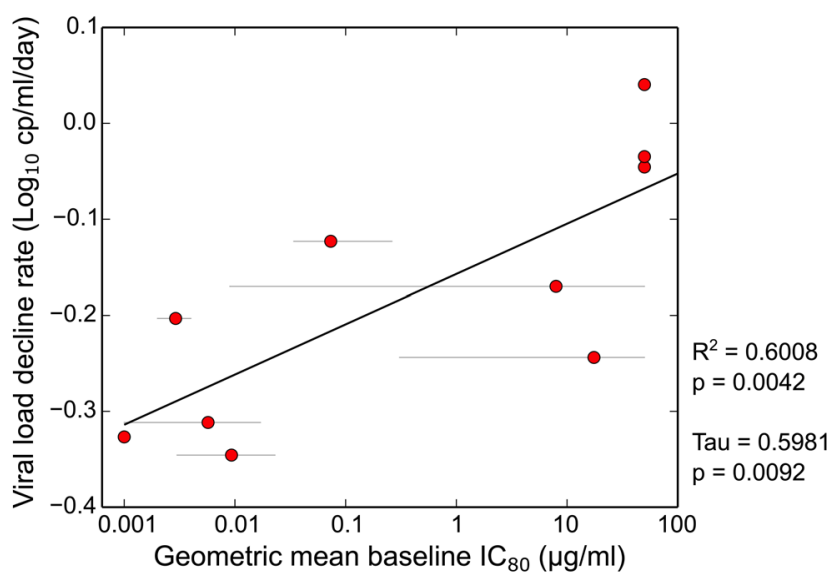

E

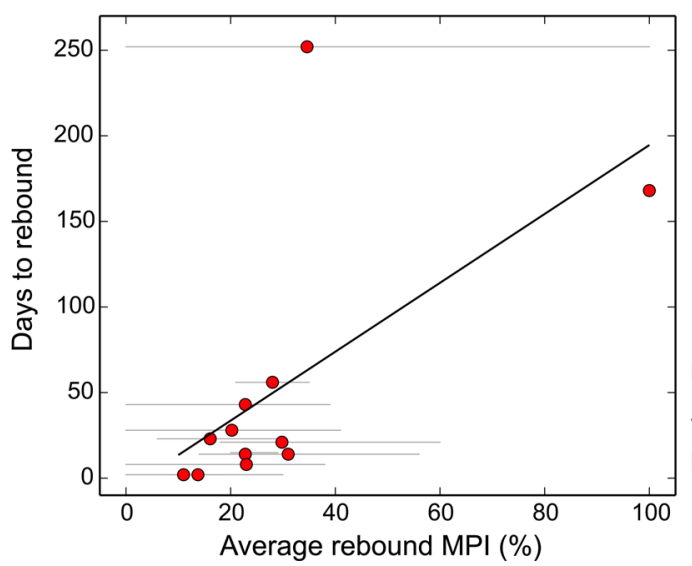

B

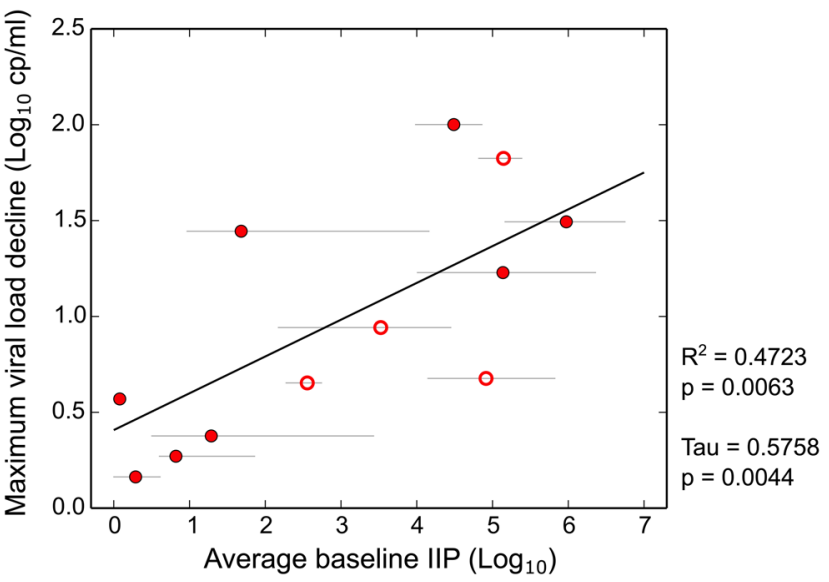

D

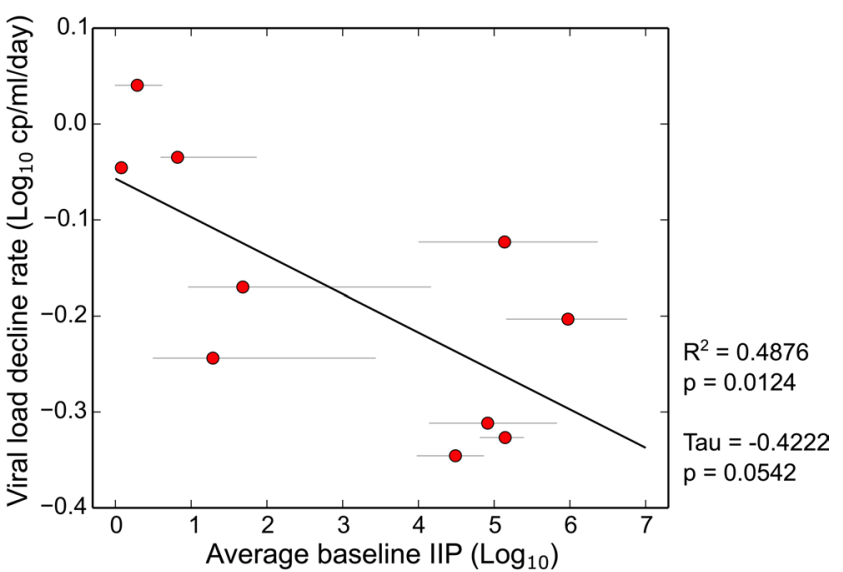

F

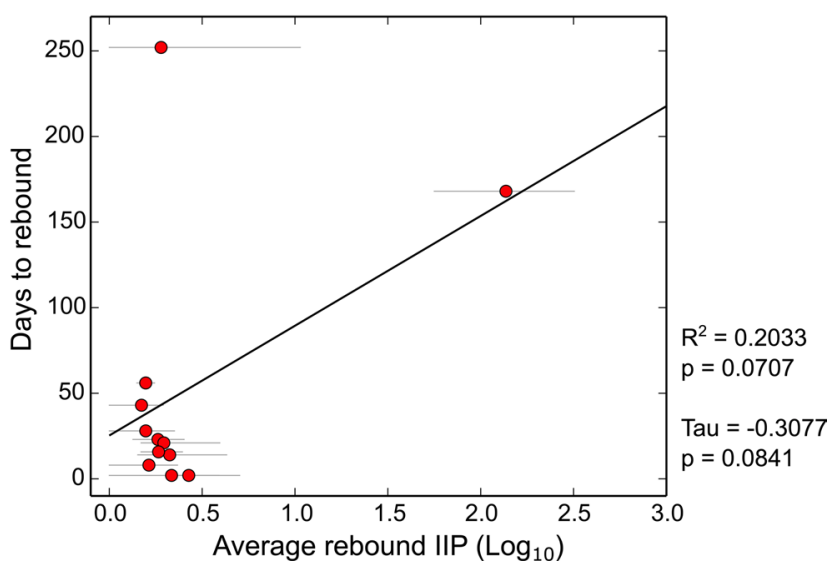

Extended Data Fig. 4 | Correlation between PGT121 neutralization and viral load kinetics. (a) Correlation between maximum viral load drop and PGT121 geometric mean IC80 titers for baseline viruses. Open symbols indicate participants whose minimum viral load was below the lower limit of quantitation (40 copies $/ \mathrm{ml}$ ). Grey lines indicate range of individual baseline virus $\mathrm{IC}_{80}$ titers. Black line is the linear regression trend line. (b) Same as ( $\mathrm{A}$ ), but using predicted average instantaneous inhibitory potential (IIP) for baseline viruses (see Methods). (c) Correlation between initial viral load decline rate and geometric mean PGT121 IC $\mathrm{C}_{80}$ titers for baseline viruses. Grey lines indicate range of $I C_{80}$ titers, black line the trend. See methods for initial viral load decline rate calculations. (d) Same as (C) but using average IIP as in (B). (e) Correlation between days to rebound and average maximum percent inhibition (MPI) of rebound viruses by PGT121. Because most rebound viruses had IC 80 above threshold ( $50 \mu \mathrm{g} / \mathrm{ml}$ ), we used MPI defined as the observed percent neutralization of each virus at $50 \mu \mathrm{g} / \mathrm{ml}$. Time to rebound was calculated as the first timepoint when the viral load post infusion returns to 0.5 Log10 copies/ml below the baseline or higher. (f) Same as (E), but using average IIP for rebound viruses. In each panel, statistics using Pearson moment correlation and Kendall Tau rank test are shown. One sided $p$-values are reported. 


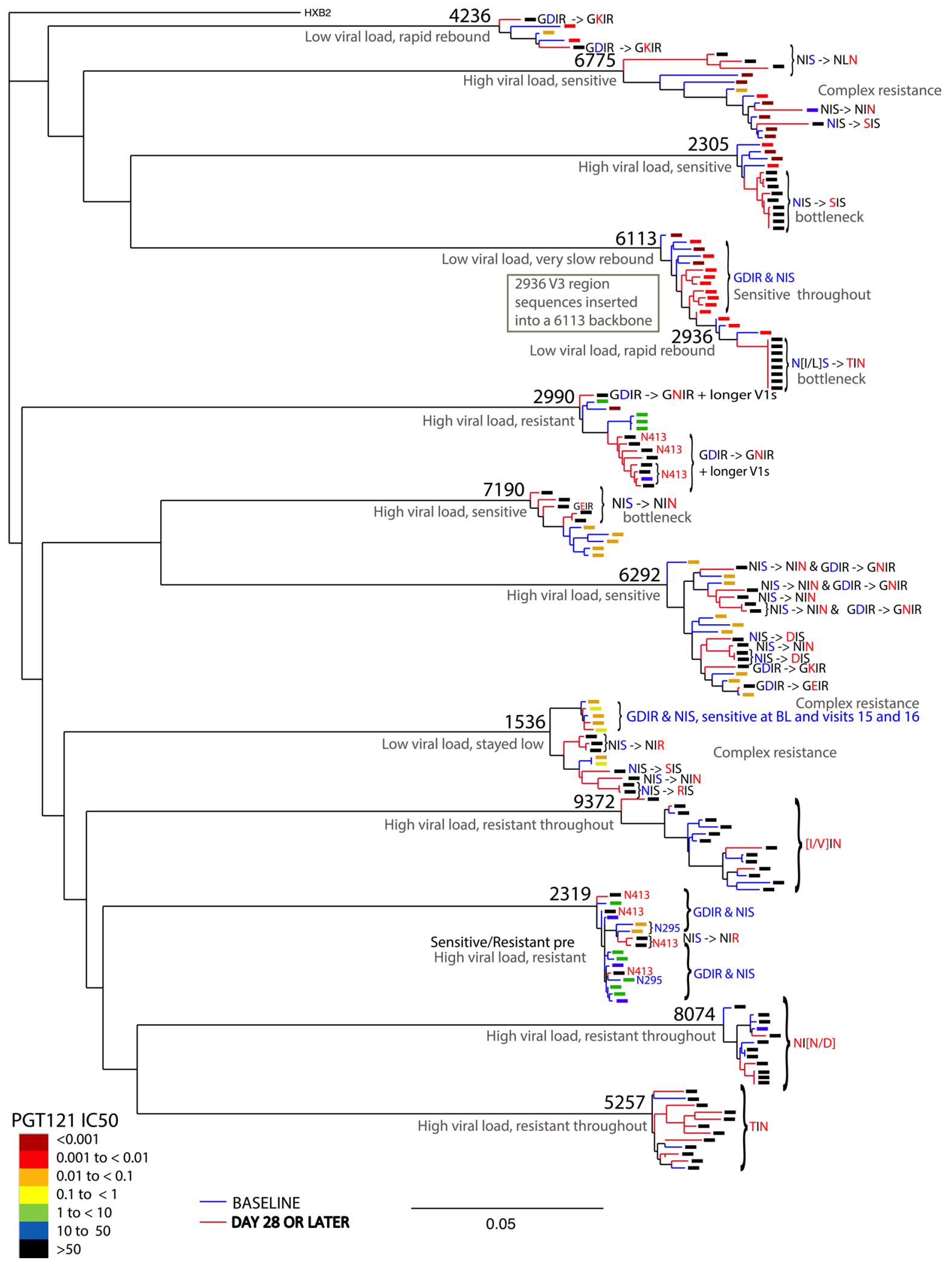

Extended Data Fig. 5 | See next page for caption. 
Extended Data Fig. 5 | Maximum likelihood phylogenetic tree of HIV-1 Env sequences isolated from participants pre- and post-PGT121 treatment. The branch colors indicate sequences sampled at baseline (blue) versus post-PGT121 treatment (red). The colored box at every leaf indicates PGT121 sensitivity as an $\mathrm{IC}_{50}$ score in a TZM.bl assay; black is undetectable, blue and green are relatively resistant, and yellow-through-red increasingly sensitive. The likely amino acids that confer PGT121 resistance mutation are indicated to the right of the branches. If a four-letter motif is indicated, it represents positions $324-$ 327 (HXB2 numbering), a conserved part PGT121 epitope; GDIR is the common consensus form and is associated with antibody sensitivity. If a three-letter motif is indicated, it refers to positions 332-334; most commonly this is a glycosylation site (NIS), and an N-linked glycan is generally critical for V3 bNAb sensitivity. Blue letters indicate PGT121 sensitive signature amino acids, red indicate resistant. The glycosylation motif can be lost by either the loss of the Asn (N) at N332, or the loss of a Thr (T) or Ser (S) at S334. D325 is the most variable position in the highly conserved GDIR motif. D325N does not always confer resistances (for example participant 6775), but is generally associated with resistance (for example participants 6292 and 2990). 
A

6292 $325,332,334$

6775
$328,330,332,334$

$$
\begin{array}{r}
2305 \\
\text { N332S } \\
\text { Loss of } 332 \text { glycan }
\end{array}
$$$$
328,330,332,334
$$

190 7190
S334N
Loss of 332 glycan
High Viral load, sensitive

"El|

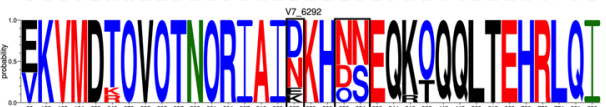

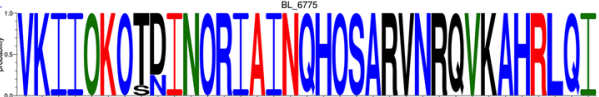

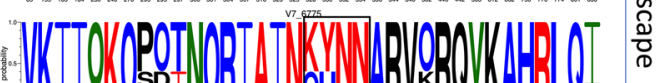

:

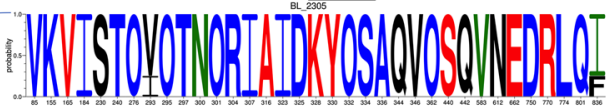



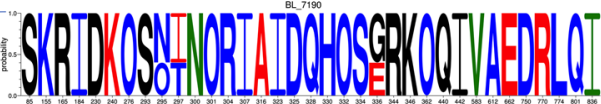



C

Low Viral load

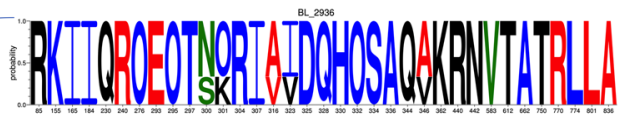

2936

N332 to N334
glycan shift

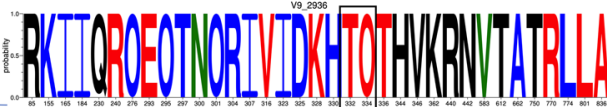

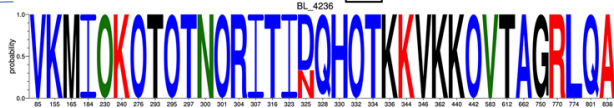

4236

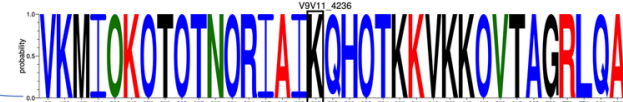



1536
332,334

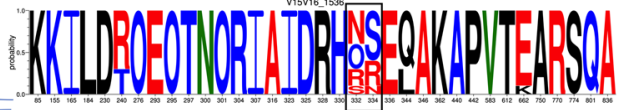

6113
B

High Viral load, resistant



or N334
glycan

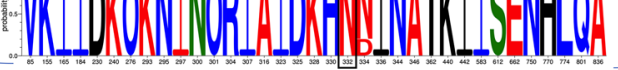

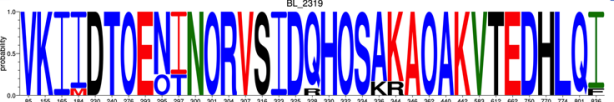

2319

:

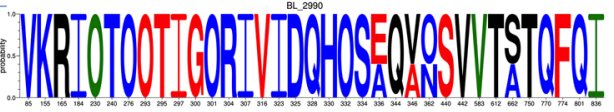

2990

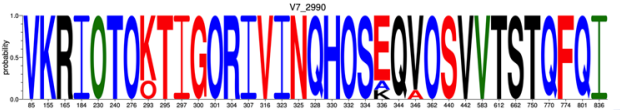

\section{.}


a PTID 6292

Time Pts

- Baseline (BL)

- Day 28 (V7)

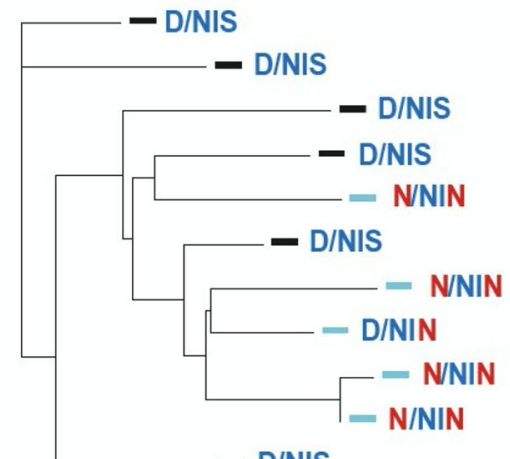

Breakpoints $\begin{gathered}\text { non-recombinant } \\ \text { recombinant } \\ \text { descendent }\end{gathered}$

IC50

IC80

\begin{tabular}{l|ll} 
& & BL.6292.H4.1_1 \\
& & BL.0.6292.C4.1_1 \\
& BL.0.6292.A4.1_1 \\
& & BL.0.6292.G4.3_1
\end{tabular}

打 回| v7.7.6292.F10.2_1

$\mathbb{\|} \mathbb{\|} \|$ BL.0.6292.D4.1_1

V7.7.6292.F12.4_1

V7.7.6292.C12.1_1

H $1 \square$

V7.7.6292.A6.1_1

V7.7.6292.A11.1_1

메 $\mathbb{M} \square$

BL.0.6292.D2.3 1

V7.7.6292.H7.4_1

$-110\|\square-\|$

V7.7.6292.G10.4_2

\&

V7.7.6292.F5.2_1

$\mathbb{\|} \| \mathbb{4}$

V7.7.6292.89.2_1

BL.0.6292.G2.1_1

BL.0.6292.F2.2_1

BL.6292.B2.1_1

V7.7.6292.C8.1_1
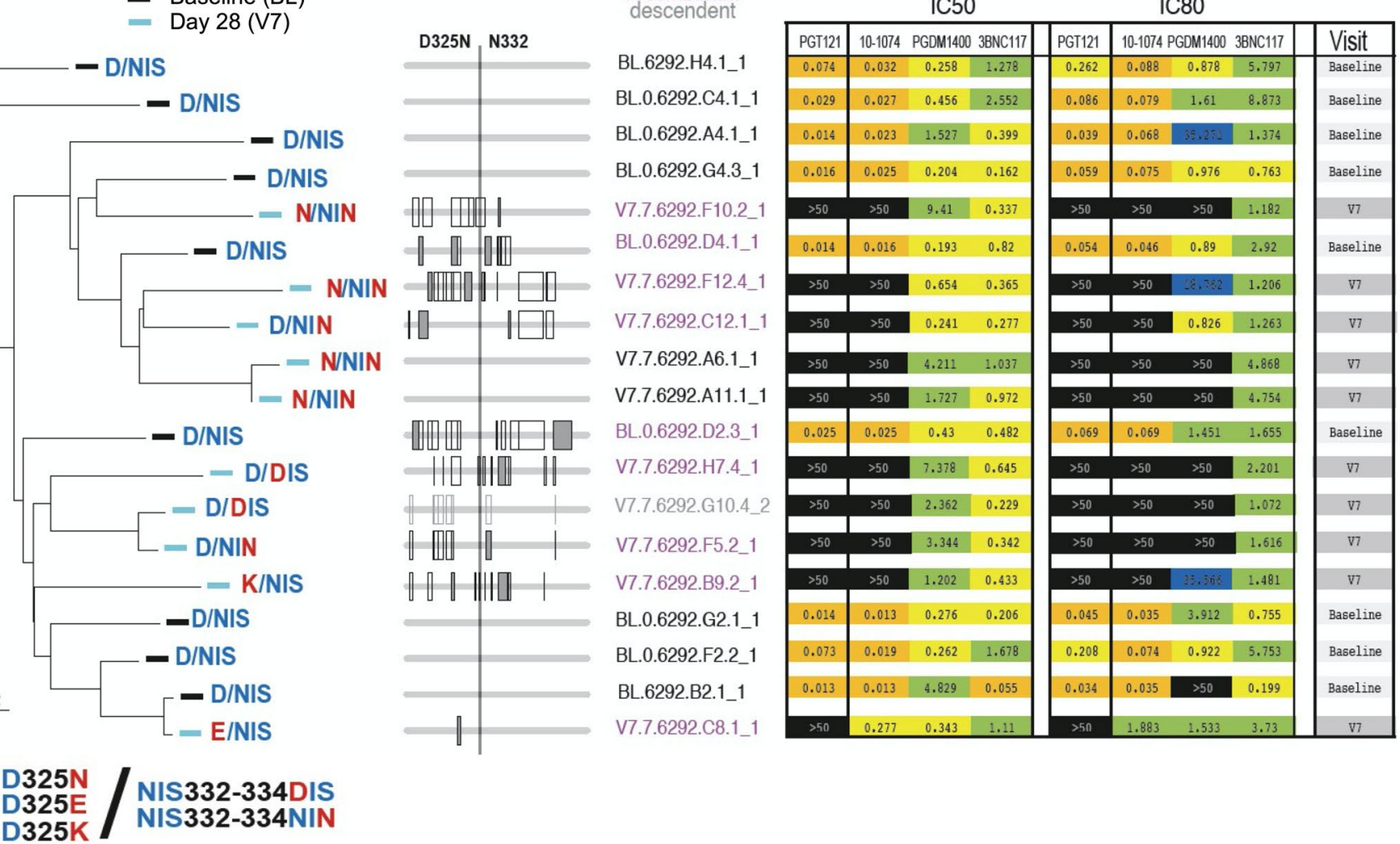

b

PTID 2990

Time Pts
- Baseline (BL)
- Day $28(\mathrm{~V} 7)$
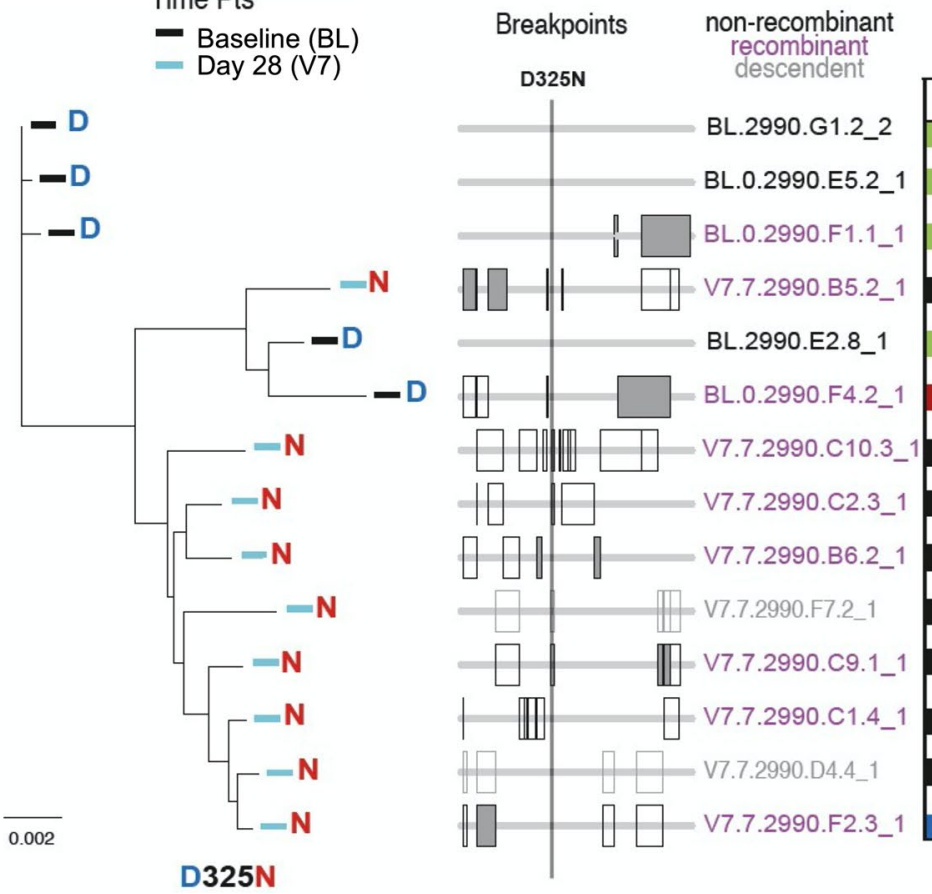

IC50

IC80

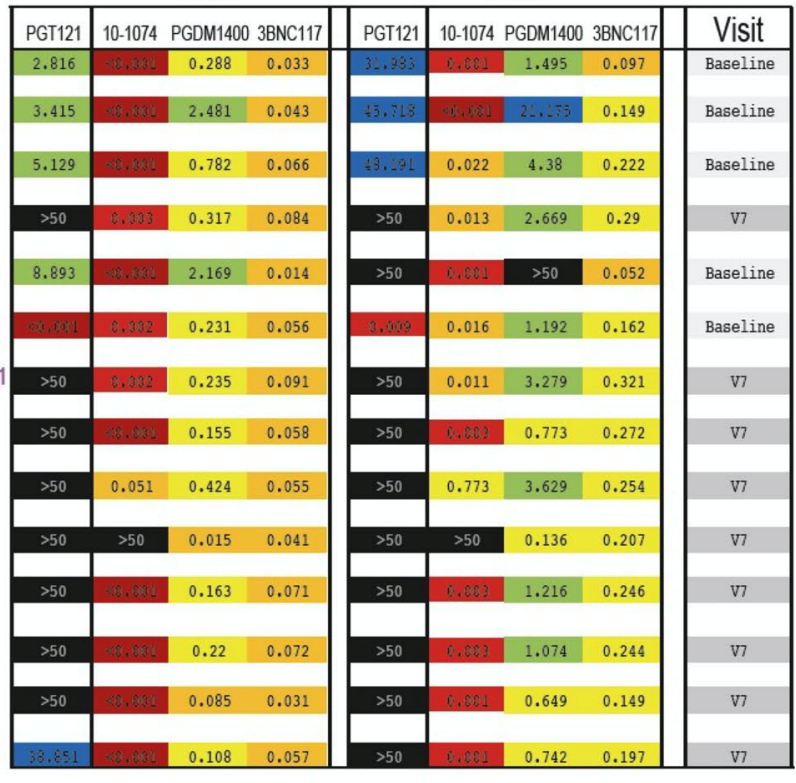

Extended Data Fig. 7 | See next page for caption. 
Extended Data Fig. 7 | Complex recombination patterns among two participants. The role of recombination in carrying forward resistant form is shown for study participants 6292 (top) and 2990 (bottom). Left panels show the phylogenetic trees with leaves labeled according to the sensitive/ resistant form they are carrying; in the middle panels the corresponding sequences (in the same order as the trees on the left) are each represented by a line, and recombinants display regions where recombination breakpoints likely occurred as rectangles (solid rectangles indicate statistically significant recombination breakpoints); corresponding neutralization data (IC50 and IC80) is shown on the right. In both study participants recombination has no impact at baseline as all forms are PGT121 sensitive. However, complex forms of resistance emerge at visit 7. In 6292, these involve D325 alone, N332 alone, and a combination of both. At visit 7, in 2 out of 2 recombination events between a sensitive and a resistant parental form, the resistant form is found in the recombinant, yet all visit 7 viruses are resistant. Either these mutations are occurring de novo on recombinant backbones, or they are resulting from recombination of parental forms that carry the mutation but have not been sampled. In 2990, recombination carries forward PGT121 resistant forms in 3 out of 3 events where the parental strains carry a sensitive and resistant form each. Of note, D325N does not confer 10-1074 resistance in 2990, neither does D325E in 6292. However, D325K, found in 6292, gives rise to resistance to both 10-1074 and PGT121. 



Extended Data Fig. 8 | Modeling the effects of PGT121 on plasma viral load. Best fit of viral dynamic model (light blue line) to measured viral load data (blue asterisks) for 6 participants. PGT121 serum concentration is shown in solid red. Horizontal dotted line indicates the viral load limit of detection. Participants 2990,6292 and 2305 shown in the top row belong to the high viral load group, with the remaining patients belonging to the low viral load group. The model follows two populations of virus, PGT121 sensitive and PGT121 resistant, denoted by the purple and orange dashed lines, respectively, while the total viral load is a solid light blue line. The level of resistance is allowed to vary among participants. For 2305, 2936, 2990, 6292 and 6113 the resistant population is predicted to pre-exist at the indicated levels. For the long-term controller 6113 the pre-existing resistant virus is predicted to be partially sensitive to the antibody and after PGT121 administration its level quickly decreases below the $x$-axis and does not reappear until after day 100. For the other long-term controller 1536, the resistant population may pre-exist at a baseline level of $10^{-3}$ copies/ml or lower or may arise by mutation. Loss of control in both 1536 and 6113 is predicted to occur due to a resurgence of resistant virus. For the periods of observation indicated, the resistant population is predicted to persist $(2305,2936$ and 2990) or to be replaced by drug sensitive virus as the antibody concentration wanes in 6292 and the long-term controllers 1536 and 6113. 

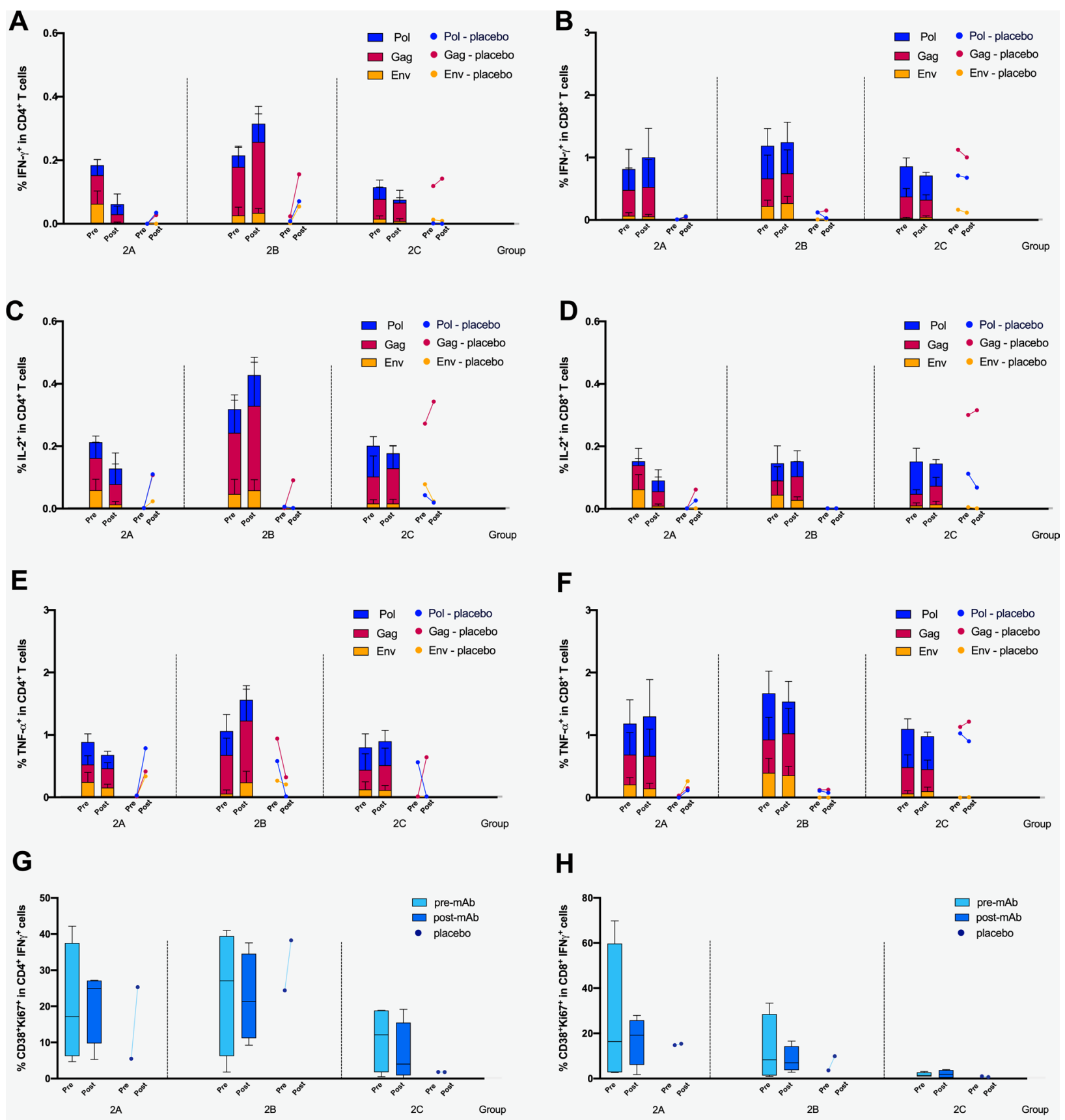

H
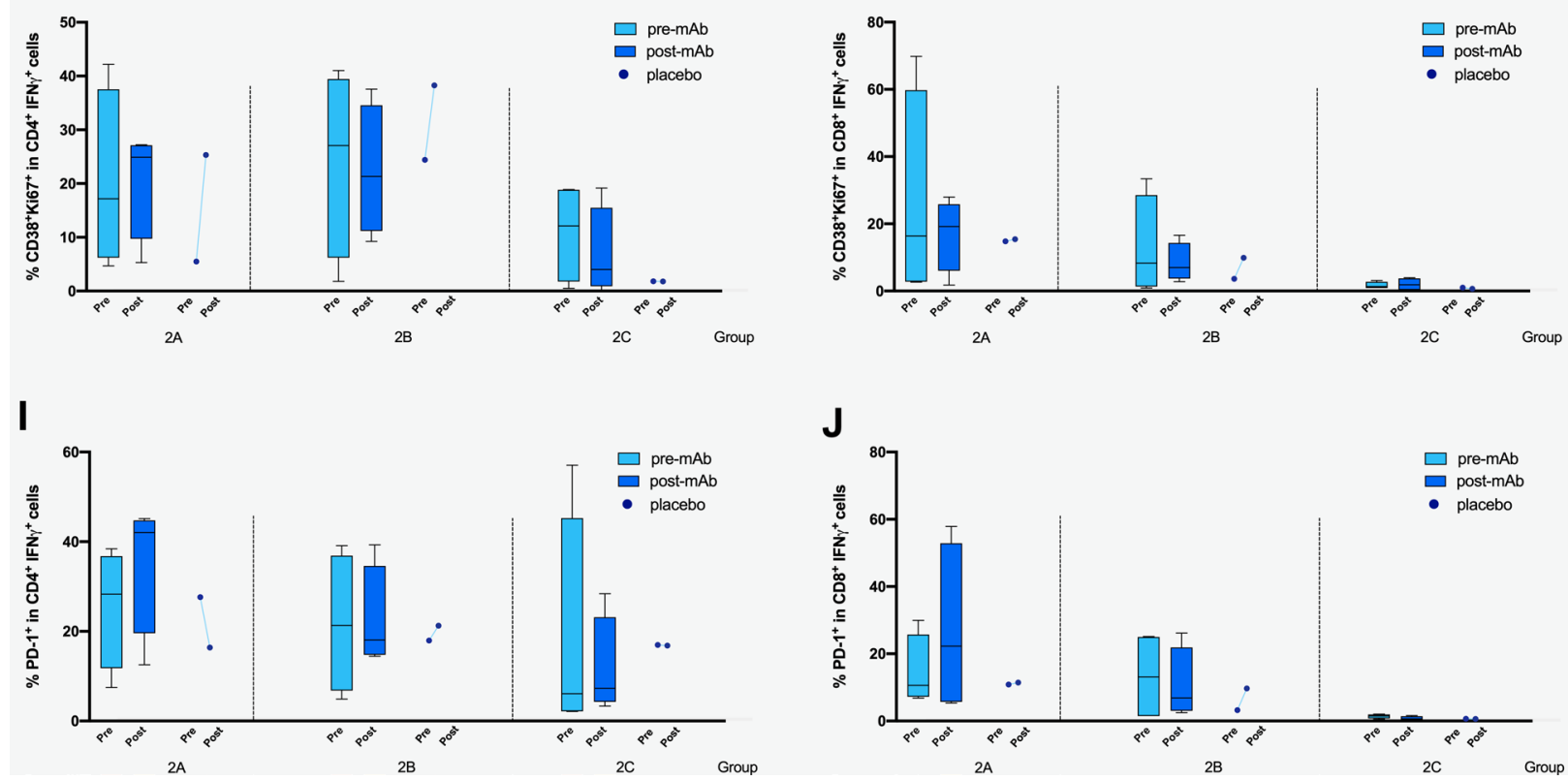

Extended Data Fig. 9 | See next page for caption. 
Extended Data Fig. 9 | Immunologic responses following PGT121 infusion in HIV-infected participants on ART. The number of IFN- $\gamma$ secreting CD4 + and CD8 $+T$ cells ( $A$ and $B$, respectively), IL-2 secreting CD4 + and CD8 $+T$ cells ( $C$ and D, respectively) and TNF-a secreting CD $4+$ and CD8 $+T$ cells ( $E$ and $\mathrm{F}$, respectively) recognizing peptide subpools across the Gag, Pol and Env protein were measured by flow cytometry in all group $2 \mathrm{~A}, \mathrm{~B}$ and $\mathrm{C}$ participants (PGT121 and placebo recipients, $\mathrm{N}=15$ ) at baseline (pre-mAb) and at day 28 (post-mAb). For panels $A-F$, data are presented as median values $+/-95 \%$ $\mathrm{Cl} ; \mathrm{n}=4$ (active) and $\mathrm{n}=1$ (placebo) for each subgroup. Frequencies of activated CD38 and Ki67 expressing IFN- $\gamma$ secreting CD4 + and CD8 $+\mathrm{T}$ cells (G and $\mathrm{H}$, respectively), and frequencies of PD- 1 expressing IFN- $\gamma$ secreting CD4 + and CD8 $+\mathrm{T}$ cells (I and J, respectively) at baseline (pre-mAb) and at day 28 (post-mAb) were assessed by flow cytometry. Boxplots display minimum, maximum, median and interquartile range; $n=4$ (active) and $n=1$ (placebo) for each subgroup. 


\section{Reporting Summary}

Nature Portfolio wishes to improve the reproducibility of the work that we publish. This form provides structure for consistency and transparency in reporting. For further information on Nature Portfolio policies, see our Editorial Policies and the Editorial Policy Checklist.

\section{Statistics}

For all statistical analyses, confirm that the following items are present in the figure legend, table legend, main text, or Methods section.

$\mathrm{n} / \mathrm{a} \mid$ Confirmed

$\bigotimes$ The exact sample size $(n)$ for each experimental group/condition, given as a discrete number and unit of measurement

$\square$ \ A statement on whether measurements were taken from distinct samples or whether the same sample was measured repeatedly

The statistical test(s) used AND whether they are one- or two-sided

Only common tests should be described solely by name; describe more complex techniques in the Methods section.

$\square$ \ A description of all covariates tested

$\square$ \ A description of any assumptions or corrections, such as tests of normality and adjustment for multiple comparisons



AND variation (e.g. standard deviation) or associated estimates of uncertainty (e.g. confidence intervals)

For null hypothesis testing, the test statistic (e.g. $F, t, r$ ) with confidence intervals, effect sizes, degrees of freedom and $P$ value noted

Give $P$ values as exact values whenever suitable.

$\bigotimes$ For Bayesian analysis, information on the choice of priors and Markov chain Monte Carlo settings

$\square$ For hierarchical and complex designs, identification of the appropriate level for tests and full reporting of outcomes

$\square$ Estimates of effect sizes (e.g. Cohen's d, Pearson's $r$ ), indicating how they were calculated

\section{Our web collection on statistics for biologists contains articles on many of the points above.}

\section{Software and code}

Policy information about availability of computer code

\section{Data collection eClinical V3.7}

Data analysis PK data was analyzed in Monolix (version 2019R1, Antony, France: Lixoft SAS, 2019). Virus neutralization modeling was done using Python Scipy Optimize package (https://docs.scipy.org/doc/scipy/reference/optimize.minimize-lbfgsb.html). Additional analysis was done with Scipy (https://docs.scipy.org/doc/scipy/reference/generated/scipy.integrate.simps.html). Viral load kinetics were modeled with Scipy as well (https://docs.scipy.org/doc/scipy-0.15.1/reference/generated/scipy.stats.mstats.theilslopes.html). Phylogenetic trees were constructed using IQ-tree and recombination analysis was performed using RAPR.

For manuscripts utilizing custom algorithms or software that are central to the research but not yet described in published literature, software must be made available to editors and



\section{Data}

Policy information about availability of data

All manuscripts must include a data availability statement. This statement should provide the following information, where applicable:

- Accession codes, unique identifiers, or web links for publicly available datasets

- A description of any restrictions on data availability

- For clinical datasets or third party data, please ensure that the statement adheres to our policy 


\section{Field-specific reporting}

Please select the one below that is the best fit for your research. If you are not sure, read the appropriate sections before making your selection.

\section{Life sciences study design}

All studies must disclose on these points even when the disclosure is negative.

Sample size The sample size for safety and tolerability analysis was 35-56 participants according to the dose escalation design. As this was an exploratory proof of concept trial and analysis was descriptive, no formal null hypothesis was tested. The frequency of moderate or greater reactogenicity events was determined and compared between groups. The frequency of SAEs judged possibly, probably or related to the IP was determined. All AEs were analyzed and, grouped by seriousness, severity and relationship to the IP (as judged by the investigator). An interim analysis of group data was carried out according to the study schema without unblinding the study to investigators or participants. At the end of the study, a full analysis was prepared. Unused and spurious data was listed separately and excluded from the statistical analysis. Missing data was excluded from the statistical analysis. (2) The sample size for pharmacokinetic (PK) analysis was 4 per dose sub-group, sufficient to provide information for the planned analyses. As this was an exploratory proof of concept trial and analysis was descriptive, no formal null hypothesis was tested. The data were fit to standard two-compartment population models using the Stochastic Approximation ExpectationMaximization (SAEM) estimation method in Monolix (version 2019RI, Antony, France: Lixoft SAS, 2019). Residual variability was estimated using an additive plus proportional error model, and models were performed separately by HIV infection status. Correlations between $\mathrm{Cl}$ and Ve were significant (Pearson's correlation coefficient p-value s 0.05 ) and were included in the models. Median-adjusted logl0-transformed weight was significantly correlated (Pearson's correlation coefficient p-value s 0.05) with Vp in the HIV-uninfected model and with Ve and Cl in the HIV-infected model and those relationships were also included in the models. Distribution and elimination half-lives were computed using the resulting model. AUC was estimated by calculating the integral of the predicted concentration-time curve from the infusion time to infinity. Peak concentration ( $\mathrm{Cmax}$ ) was computed as the maximum observed concentration. Summary descriptive results of individual estimates of PK parameters, including AUC, Cmax, TI/2, and clearance results were reported by dose cohort. Correlation between PK and reported safety and pharmacodynamic outcomes were also explored parameters in order to examine exposure-effect relationships. (3) The frequency and levels of anti-PGT121 antibodies were calculated and tabulated. (4) The sample size for virologic analysis in Group 3A was 9 participants. As this was an exploratory proof of concept trial and the analysis was descriptive, no formal null hypothesis was tested. The primary outcome for this analysis was defined as change in logl0 viral load between Day 0 (day of infusion) and Day 7. No placebo participants were enrolled as part of this design. The statistical test performed was the Signed-rank test, which incorporated the "shift" parameter of -0.9 $\log \mid 0$. An evaluation of potential harm (increased viral load) was also performed with the Signed rank test; this test examined the null hypothesis of no change in viral load (a shift of $0.0 \log \mid 0$ following IP administration) against the one-sided alternative hypothesis that the viral load is increased following IP administration. Each efficacy test was performed at the level a $=0.05$. Each test for harm was performed at level $2 \mathrm{a}=0.10$, in order to provide additional sensitivity to detect potential harm. (5) The sample size for antiviral activity in group 3D was up to 6 participants. As this was an exploratory proof of concept trial and the analysis was descriptive in this population, no formal null hypothesis was tested. No efficacy endpoints were tested in Groups 3D as participants were HIV-infected with low viral loads at baseline (102 - 2x103 copies $/ \mathrm{ml}$ ). Immunologic and virologic endpoints were determined as described in Protocol Version 8.0 Section 4.1

Data exclusions None

$\begin{array}{lll}\text { Replication } & \text { This section does not apply to our study which was a clinical trial with unique participants who could not be replicated. There were, however, }\end{array}$ 48 participants enrolled who received some of the same interventions as outlined below.

Randomization In Part 1, eligible participants were enrolled into the lowest dose sub-group (Group IA and 2A) first according to their HIV-serostatus and in parallel. Participants in each sub-group were identified by a unique study identification number. Participants were randomized according to the randomization schedule prepared by the statisticians at the Data Coordinating Center (DCC) prior to the start of the study. Participants were automatically assigned a specific allocation number as they were enrolled into the data entry system. The 5 participants in each sub-group were randomized in a ratio of four PGT121 recipients to one placebo recipient. At each dose level in Part 1, IP administration was separated by at least 24 hours for each of the first 3 participants. Randomization ensured at least 2 participants received active product and were observed for at least 24 hours before administration to additional participants. monitoring the safety and laboratory assay results) and participants were blinded with respect to the allocation of investigational product (IP). A site pharmacist was unblinded for the purposes of preparing study product. Blinded participants were informed about their assignment (product/placebo) at study completion, once the data was locked. Group 1 and Group 2 were unblinded separately after the last participant in the respective groups completed study participation. As PGT121 and placebo (saline) looked identical in the infusion bag, no masking was required.

\section{Reporting for specific materials, systems and methods}

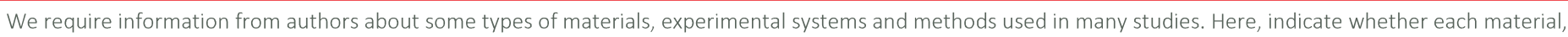

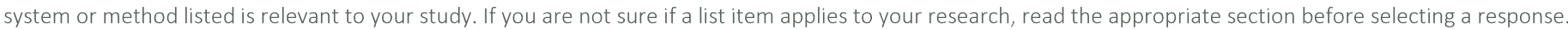




\begin{tabular}{l|l}
\hline$n / a$ & Involved in the study \\
\hline & $\bigotimes$ Antibodies \\
$\square$ & $\square$ Eukaryotic cell lines \\
$\square$ & $\square$ Animals and other organisms \\
$\square$ & $\bigotimes$ Human research participants \\
$\square$ & $\bigotimes$ Clinical data \\
$\searrow$ & $\square$ Dual use research of concern
\end{tabular}

\section{Antibodies}

Antibodies used

Drug Product: PGT121. Althea/Catalent Lot Number 1-FIN-2442. Date of Fill: 18AUG2016. The product was produced by Catalent Pharma Solutions, Madison WI, USA and Ajinomoto Althea, San Diego, CA in accordance with GMP.

Validation

All detailed product information on PGT121 is available in the Investigator's Brochure which was submitted for this trial under IND 126807, including specifically Section 3, "Physical, Chemical and Pharmaceutical Properties." The IB is available upon request from $\mathrm{IAVI}$, the sponsor.

\section{Human research participants}

\section{Policy information about studies involving human research participants}

Population characteristics

ales and females aged 18-50 years old who were willing to maintain low risk behavior for HIV infection (Group 1); HIVinfected males and females aged 18-65 years old on a stable antiretroviral regimen with HIV-1 RNA plasma level <50 copies/ $\mathrm{ml}, \mathrm{CD} 4$ cell count? 300 cells/ $\mu \mathrm{L}$, and no history of AIDS-defining illness within the previous 5 years (Group 2); and HIVinfected males and females aged 18-65 years old, not on antiretroviral therapy for $>6$ months with detectable HIV- 1 viral load between 100 to 2,000 copies/ml and 2,000 to 100,000 copies/ml (as per protocol), CD4 cell count> 300 cells/ $/ \mathrm{L}$, and no history of AIDS-defining illness within the previous 5 years (Group 3).

Recruitment

Adult male and female participants were recruited through in-clinic referrals, information presented to community organizations, hospitals, colleges, other institutions and/or advertisements to the general public or from existing cohorts. The information distributed contained information about the trial and contact information for the site. Study staff members also attended events related to public health, HIV/AIDS, sexual health, and other topics as appropriate. Because participants were recruited from North America, HIV sequence diversity was biased towards clade B viruses that may be less susceptible to PGT121 compared to other clades. For our HIV-negative population, recruits were from the Boston area and were relatively white compared to the general population of HIV-infected patients; this may limit generalisability to other populations.

Ethics oversight BIDMC Institutional Review Board, the OIC Institutional Review Board, and the HART Committee for the Protection of Human Subjects

Note that full information on the approval of the study protocol must also be provided in the manuscript.

\section{Clinical data}

Policy information about clinical studies

All manuscripts should comply with the ICMJE guidelines for publication of clinical research and a completed CONSORT checklist must be included with all submissions.

Clinical trial registration NCT02960581

Study protocol

The protocol and amendments were provided as an appendix to the manuscript.

Data collection

Ninety-eight participants were screened, and 50 were found to be ineligible or excluded for other reasons (Fig. 1). The first participant was enrolled on Jan 17, 2017, the last participant was enrolled on Jan 22, 2019, and the last study visit was completed on Jul 8, 2019. Part 1 of the study was a single-center, randomized, double-blind, dose-escalation, placebo-controlled trial of PGT121 in HIV-uninfected adults and HIV-infected adults on antiretroviral therapy (ART) at Beth Israel Deaconess Medical Center (BIDMC), Boston MA. Part 2 of the study was a multi-center, open-label trial of PGT121 in viremic HIV-infected adults not on ART at three sites: BIDMC, Boston MA, Orlando Immunology Center (OIC), Orlando FL, and Houston AIDS Research Team (HART), McGovern Medical School at The University of Texas Health Science Center at Houston TX.

Outcomes

The primary endpoints were, for safety and tolerability: (1) proportion of participants with moderate or greater reactogenicity (e.g., solicited AEs) for 3 days following IV infusion or SC injection of PGT121 mAb, (2) proportion of participants with moderate or greater and/or PGT121 mAb-related unsolicited AEs, including safety laboratory (biochemical, hematological) parameters, following IV infusion or SC injection of PGT121 mAb for the first 56 days post administration of IP, and (3) proportion of participants with PGT121 mAb-related SAEs throughout the study period. The primary endpoints, for pharmacokinetics, were elimination half-life ( $t \mid / 2)$, clearance $(\mathrm{CL} / \mathrm{F})$, volume of distribution $(\mathrm{VZ} / \mathrm{F})$, area under the concentration decay curve (AUC), and impact of viral load and/or ART on PGT121 disposition (elimination half-life (tl/2), clearance (CL/F), volume of distribution (Vz/F), and total exposure. The primary endpoint for antiviral activity among viremic HIV-infected adults not on ART was the change in plasma HIV-1 RNA levels from baseline 
(mean of pre-entry and entry values). The secondary endpoints were change in serum anti-PGT121 antibody titers from baseline, change in CD4+ T cell count and frequency compared to baseline as measured by single platform flow cytometry, and development of HIV-1 sequence variations in epitopes known to result in reduced PGT121 mAb neutralization susceptibility. Additional exploratory assessments could include, but were not limited to, the following: HIV-specific

IgG/IgA binding responses by ELISA, HIV-specific cellular immune responses by ELISPOT, HIV-specific antibody function by ADCC, $A D C P$, and ADCVI assays, resistance mutations to current ARVs, PGT121 mAb levels in mucosal secretions, changes in total HIV-1 DNA and 2-long terminal repeat (LTR) circular HIV-1 DNA in resting or total CD4 T cells and in vitro neutralization of HIV isolates with participant's serum post administration of PGT121 mAb. Available samples from time points during the optional LTE phase were also used for determination of

long-term durability of the immune responses. The primary endpoints for safety, tolerability and pharmacokinetics were changed in Protocol Version 6.0 to nclude evaluate of both IV and SC routes, after the addition of sub-group 1D to the trial design. All primary endpoints were also changed in Protocol Version 8.0 to include evaluation of data from participants enrolled in the optional LTE phase. 